\title{
Rolling stock scheduling with maintenance requirements at the Chinese High-Speed Railway
}

Zhong, Qingwei; Lusby, Richard Martin ; Larsen, Jesper; Zhang, Yongxiang; Peng, Qiyuan

Published in:

Transportation Research Part B: Methodological

Link to article, DOI:

10.1016/j.trb.2019.05.013

Publication date:

2019

Document Version

Peer reviewed version

Link back to DTU Orbit

Citation (APA):

Zhong, Q., Lusby, R. M., Larsen, J., Zhang, Y., \& Peng, Q. (2019). Rolling stock scheduling with maintenance requirements at the Chinese High-Speed Railway. Transportation Research Part B: Methodological, 126, 24-44. https://doi.org/10.1016/j.trb.2019.05.013

\section{General rights}

Copyright and moral rights for the publications made accessible in the public portal are retained by the authors and/or other copyright owners and it is a condition of accessing publications that users recognise and abide by the legal requirements associated with these rights.

- Users may download and print one copy of any publication from the public portal for the purpose of private study or research.

- You may not further distribute the material or use it for any profit-making activity or commercial gain

- You may freely distribute the URL identifying the publication in the public portal 


\section{Rolling stock scheduling with maintenance requirements at the Chinese}

\section{High-speed railway}

Qingwei Zhong ${ }^{\mathrm{a}, \mathrm{b}}$, Richard M. Lusby ${ }^{\mathrm{b}}$, Jesper Larsen ${ }^{\mathrm{b}}$, Yongxiang Zhang $^{\mathrm{a}, \mathrm{c}}$, Qiyuan Peng ${ }^{\mathrm{a}}$

${ }^{\text {a }}$ School of Transportation \& Logistics, Southwest Jiaotong University, Chengdu, China

${ }^{\mathrm{b}}$ Department of Engineering Management, Technical University of Denmark, Produktionstorvet, building 424,

2800 Kgs. Lyngby, Denmark

${ }^{\mathrm{c}}$ School of Sustainable Engineering and the Built Environment, Arizona State University, Tempe, AZ 85281, USA

E-mail addresses: qingweizhong@my.swjtu.edu.cn (Qingwei Zhong), rmlu@dtu.dk (Richad M. Lusby),

jesla@dtu.dk (Jesper Larsen), bk20100249@my.swjtu.edu.cn(Yongxiang Zhang),

qiyuan-peng@swjtu.edu.cn (Qiyuan Peng) 


\begin{abstract}
Efficient rolling stock schedules have always been the focus of railway companies. At the Chinese High-Speed Railway, rolling stock scheduling is usually done manually by dispatchers. Such an approach often makes it difficult to quickly obtain an optimal rolling stock schedule that can be used to operate the proposed timetable and which is also feasible with respect to the rolling stock maintenance requirements. In this paper, we consider the problem of rolling stock scheduling with maintenance requirements and propose a heuristic approach that decomposes the problem into two main stages. In the first stage, the maintenance restrictions are ignored and a conventional Mixed Integer Programming model is used to generate multiple candidate rolling stock schedules. In the second stage, we check to see whether any of the candidate rolling stock schedules are feasible when maintenance restrictions for the individual rolling stock units are also considered. Checking a candidate rolling stock schedule can be performed using an assignment problem. To provide some structure to the search, candidate rolling stock schedules generated in the first stage are sorted in descending order according to their objective values. The two stages can be iteratively performed to avoid generating all schedules in the first stage before proceeding to the second stage. We analyze the performance of the proposed approach on real-life instances provided by the Chinese High-Speed Railway. These instances focus on the railway network within the Zhengzhou Group, which is one of the busiest transportation networks in China. Comparisons with the manual approach that is currently used in practice show that the proposed approach yields far superior schedules. The optimized schedules improve rolling stock efficiency and lead to a reduction in operating cost of approximately $10.5 \%$.
\end{abstract}

Keywords: high-speed railway; rolling stock scheduling; maintenance requirements 


\section{Introduction}

In China, the railway network, as in many other countries, forms an integral part of the transportation infrastructure. The Chinese High-Speed Railway (CHSR) network in particular is an attractive service that provides fast, direct connections to many of the country's large cities. In 2017, the CHSR network measured 25,000 km in total track length, connected 295 stations, and serviced 1.73 billion passengers. At that time it already accounted for more than 66 percent of the world's HSR network. According to the official Medium and Long Term Strategic Development Plan of the CHSR, there are ambitious plans to increase the network further to $30,000 \mathrm{~km}$ by 2020 and to 38,000 $\mathrm{km}$ by 2025 (Wangshu Luo, 2018). The overall aim is to connect all Chinese cities with populations over half a million by the CHSR network.

The CHSR has a fleet of 2,600 trains. On any given day, approximately 2,300 of these are in operation and collectively cover around 4,500 timetabled services. Twenty four depots are scattered throughout the CHSR network, and these are the locations where rolling stock maintenance can be performed. All rolling stock units must comply with certain maintenance requirements (e.g., time and distance) to ensure the safety of the system. High-speed rolling stock units require advanced technical equipment which is extremely expensive to purchase and maintain and accounts for a significant part of the railway investment in China. Therefore, from a practical perspective, efficient utilization of available rolling stock is a question of paramount importance and motivates this research. Having received relatively limited attention in the literature, the topic of generating maintenance feasible rolling stock schedules is also interesting from a theoretical perspective.

The timetable for the CHSR is revised annually (however, there may also be slight adjustments during peak periods and holidays, e.g., Chinese New Year). Usually, when the timetable requires modification, all dispatchers gather to devise a new rolling stock schedule. Depending on the complexity of the revisions and the experience of the dispatchers, a complete schedule can take several days to produce. Manually constructed schedules not only consume significant amounts of manpower and time, but they may not even satisfy all of the maintenance requirements. A decision support system to optimize this process will clearly be beneficial in alleviating some of the scheduling pressure and it will also help dispatchers by providing maintenance feasible rolling stock schedules with guaranteed quality. This further motivates the work of this paper.

We focus on modeling and solving the practical problem of including maintenance restrictions when generating rolling stock schedules. The proposed methodology can be applied to any passenger railway company; however, we exploit the fact that the CHSR is comprised of long stretches of tracks with limited locations where (de)coupling can occur. We develop a two-stage heuristic approach to address the problem. In the first stage, maintenance considerations are removed, and the arc flow model based on Fioole et al. (2006) is used to generate multiple rolling stock schedules. For each of the found schedules, the set of all possible anonymous individual rolling stock trip sequences is generated. In the second stage, a MILP assignment model is solved for each candidate rolling stock schedule from the first stage. Each MILP assignment problem considers a specific set of trip sequences and attempts to assign an 
individual trip sequence to each physical rolling stock unit, while taking the specific maintenance limits of each unit into consideration. The two stages are embedded in an iterative loop if no maintenance feasible rolling stock schedule exists in the first stage. To provide some structure to the search, candidate rolling stock schedules are considered in order of worsening objective values. Through the available lower bound information, we are able to determine the quality of any found maintenance feasible rolling stock schedule. We test the performance of the proposed methodology on the railway network defined within the Zhengzhou Group, an independent sub-network of the CHSR. This network is located in central China and extends to all directions of China. Numerical results indicate that the schedules provided by the proposed approach significantly outperform the schedules generated manually by the dispatchers.

The main contribution of this paper is in the development of a novel two-stage approach for solving the rolling stock scheduling problem with maintenance requirements. While the core mathematical model used for generating candidate rolling stock schedules has been proposed previously, its coordination with an assignment problem to detect maintenance feasible rolling stock schedules has, to the best of the author's knowledge, not. The structured way in which candidate rolling stock schedules are considered guarantees that the quality of the found rolling stock schedule can be determined. The proposed approach is benchmarked on a number of instances from the CHSR and shows promising results where optimized and maintenance feasible schedules are obtained in minutes. Unlike previous research in this area, we also focus on including more than one maintenance requirement per rolling stock unit. The two stage approach enables multiple restrictions to be easily incorporated.

In addition to this, the paper also makes some minor contributions. Firstly, it demonstrates the applicability in practice of the proposed methodology. The performance of the developed algorithm is analyzed on two actual instances that have been provided by the CHSR, and all results have been verified by dispatchers at the CHSR. The optimized schedules not only improve rolling stock efficiency, but also do so with a significantly lower operating cost.

Secondly, the paper provides a broad overview of rolling stock scheduling at the CHSR. Most of the literature available on rolling stock scheduling focuses on European passenger railway companies. This paper provides another perspective by highlighting the similarities with and the differences to the CHSR.

The CHSR is currently facing challenges in relation to the replacement of rolling stock units and the inefficient use of some units. This paper provides insight into how the CHSR operates and will hopefully motivate further research in this area. The proposed approach provides optimized rolling stock schedules that can be produced quickly and which can be readily understood by dispatchers.

The rest of this paper is structured as follows: In Section 2 we give a brief overview of related literature in the area of rolling stock scheduling with maintenance requirements. A formal problem description is given in Section 3. Section 4 describes the proposed mathematical models, along with our solution approach. The results of applying the proposed methodology to real-life instances from the CHSR are presented in Section 5. In this section we also make a direct comparison to the solution provided by the dispatchers. Finally, conclusions and directions for future research are discussed in Section 6.

\section{Literature overview}

The rolling stock scheduling problem is a classical and important problem in the 
operational planning and execution of railway operations (for a general overview of planning rail operations see e.g., Lusby et al. 2011). Properly executed, it can have a profound impact on not only the quality of the planning but also on the cost of the operations. Therefore, it has been the subject of a number of research papers over the last couple of decades. One of the first Operations Research (OR) based approaches reported is Ziarati et al (1997), where an arc-based multi-commodity flow formulation is used to assign locomotives to train-segments. Due to its size, the model is solved using Dantzig-Wolfe Decomposition. The early papers Cordeau (2000) and Cordeau et al. (2001) also use an arc-based multi-commodity flow formulation for assigning locomotives as well as rail car departures. In a number of papers the main objective is to minimize the total cost, often guided by the number of train units that are necessary to execute the plan. This is the case in Brucker et al. (2003), Abbink et al. (2004), Alfieri et al. (2006), Fioole et al. (2006), and Peeters and Kroon (2008). It is worth mentioning that Abbink et al. (2004) rely on an assignment-based approach instead of the arc-based multi-commodity flow approach. Notable contributions include Alfieri et al. (2006) and Fioole et al. (2006) as these are the first papers to include train compositions in the developed approaches. Brucker et al. (2003) include constraints on the available depot capacity. More recently, path-based multicommodity flow approaches have appeared (see e.g., Thorlacius et al. (2015) and Haahr et al. (2016)). Instead of modelling individual movements, these approaches model sequences of movements of train units. Cacchiani et al. (2013) adopts yet another approach, using a Lagrangian relaxation based heuristic to solve the problem via a sequence of assignment problems.

Among the first papers to consider maintenance, are Maroti and Kroon (2005) and Maroti and Kroon (2007), where a fixed rolling stock schedule is given at first as the basis of a directed graph and then the models are developed to modify the schedule by inserting maintenance tasks into this directed graph so that the units which close to its mileage maintenance restriction can arrive at a maintenance facility in time. The proposed approach sometimes may not find a feasible solution, and planners need to manually intervene in order to obtain a maintenance feasible schedule. Wang et al. (2010) also considers distance-based maintenance routing in an optimization-based approach using Dantzig-Wolfe Decomposition. Ohno et al. (2012) proposes a very similar decomposition approach for handling the rolling stock assignment problem with time-based maintenance constraint. The proposed approach is tested on a case study from a Japanese railway company. A heuristic based on column generation is also described in Li et al. (2014). A path-based multi-commodity flow formulation is used to route trains with distance-based maintenance restriction. The method developed is applied to a case in France.

Giacco et al. (2014) consider the punctuality of maintenance. The main constraints are used to keep timely maintenance for every rolling stock unit. The problem is modelled as a minimal cost Hamiltonian cycle problem. A similar approach is presented in Nishi et al. (2017), but instead of using a commercial solver to solve the mathematical model, the authors present a column generation based heuristic approach. Borndörfer et al. (2016) provide a solution approach based on modelling the rolling stock problem using hyper-graphs. Time and distance maintenance requirements and infrastructure capacity are also taken into consideration. It needs a relatively long computation time to solve a real-life problem. Finally, in Lai et al (2015) and Lai et al (2017), the rolling stock routing problem for one high speed railway line (the Taiwanese High-Speed rail) is solved. The approaches consider regulatory constraints as well 
as time and distance maintenance requirements. Lai et al (2015) is based on a hybrid heuristic approach, while Lai et al (2017) proposes an exact approach for the problem. Due to the timeliness of the rescheduling problem, fast-solving algorithms are often developed. For the rescheduling problems, we refer readers to Nielsen et al. (2012), Wagenaar et al. (2017), Haahr et al. (2016) and Lusby et al. (2017).

While much literature already exists on rolling stock scheduling, and to some extent the incorporation of maintenance requirements, but very few include maintenance restrictions when identifying the rolling stock schedule. Some of the approaches in the literature can indeed be used by the CHSR, e.g., Haahr et al. (2016). In Haahr et al (2016), a column generation approach is described for solving the rolling stock schedule. The model determines trip sequences for unit types. By directly dealing with trip sequences, the model can constrain the distance and running time of unit types. However, if one wishes to constrain trip sequence distance and time for each individual unit, then the column generation approach results in one sub-problem for every unit. Furthermore, Haahr et al. (2016) show the performance of the approach when only considering remaining mileage. In our work we have two restrictions to consider, and this further complicates the column generation procedure. Comparisons between the column generation approach applied to rolling stock scheduling problem without maintenance show that it is inferior to the arc-flow model of Fioole et al. (2006). Therefore, we view the model of Fioole et al. (2006) as a good starting point. It is difficult, however, to directly include maintenance restrictions in this model, leading us to the two-stage method. Wagenaar et al. (2017) also address maintenance, but from a rescheduling perspective, i.e., retaining maintenance appointments when rescheduling disrupted rolling stock units. We are interested in considering maintenance restrictions when determining rolling stock schedules.

Compared to the European railway, the rolling stock service of a specific line in the CHSR railway network is determined by the control range of a sub-company belonging to the CHSR. Within the control range of the sub-company, usually, there are not many stations that can be used for composition changes. This allows us to enumerate all possible trip sequences using the results found by the arc-flow model in a short time. The proposed approach also offers a solution pool that can be reused when the timetable, the total number of rolling stock units and depot balance are kept. In other words, if the initial states in terms of mileage and running time change for each of the units, we only need to consider the second stage of our approach.

\section{Problem description}

In this section, we provide a more formal description of the rolling stock scheduling problem that we address. In general, the rolling stock scheduling problem is a complex optimization problem (see e.g., Nielsen et al. (2012)). We introduce some basic concepts and the associated terminologies, and we state any assumptions that we make. Where relevant, specific operational processes at the CHSR are mentioned.

The rolling stock scheduling problem assumes that a timetable is available. The services specified in the timetable are defined by a set of trips. Each trip specifies the movement of a train between two stations at a specific time. Thus, a trip is formally defined as having a departure station, a departure time, an arrival station, and an arrival time. The number of passengers on each trip can be forecast according to the historical passenger flow data. It is therefore possible to specify a minimal number of units required on each trip. 
Fundamentally, the rolling stock scheduling problem involves assigning so-called compositions to a given timetable. A composition refers to a specific set of rolling stock types coupled together in a specific order. Rolling stock types differ in their physical characteristics (e.g., length, number of seats etc.) and capabilities (e.g. speed). A railway company usually has multiple rolling stock types and several units of each type. Each rolling stock unit is comprised of a number of cars. A rolling stock unit is the shortest vehicle that can be assigned; i.e., it cannot be decoupled further. Two units of the same type typically differ in, among other things, their mileage and since maintenance.

At the CHSR, there are several different types of rolling stocks available. Some of the older types cannot be coupled to or decoupled from other units after leaving the depot. This means that these types of rolling stocks can only form one possible composition, having a fixed number of cars. The newer rolling stock types, however, can form multiple compositions as they are able to be coupled and decoupled to other units. Operational requirements at the CHSR stipulate that only units of the same type can be coupled. Figure 1 illustrates four different unit types and seven possible compositions at the CHSR.

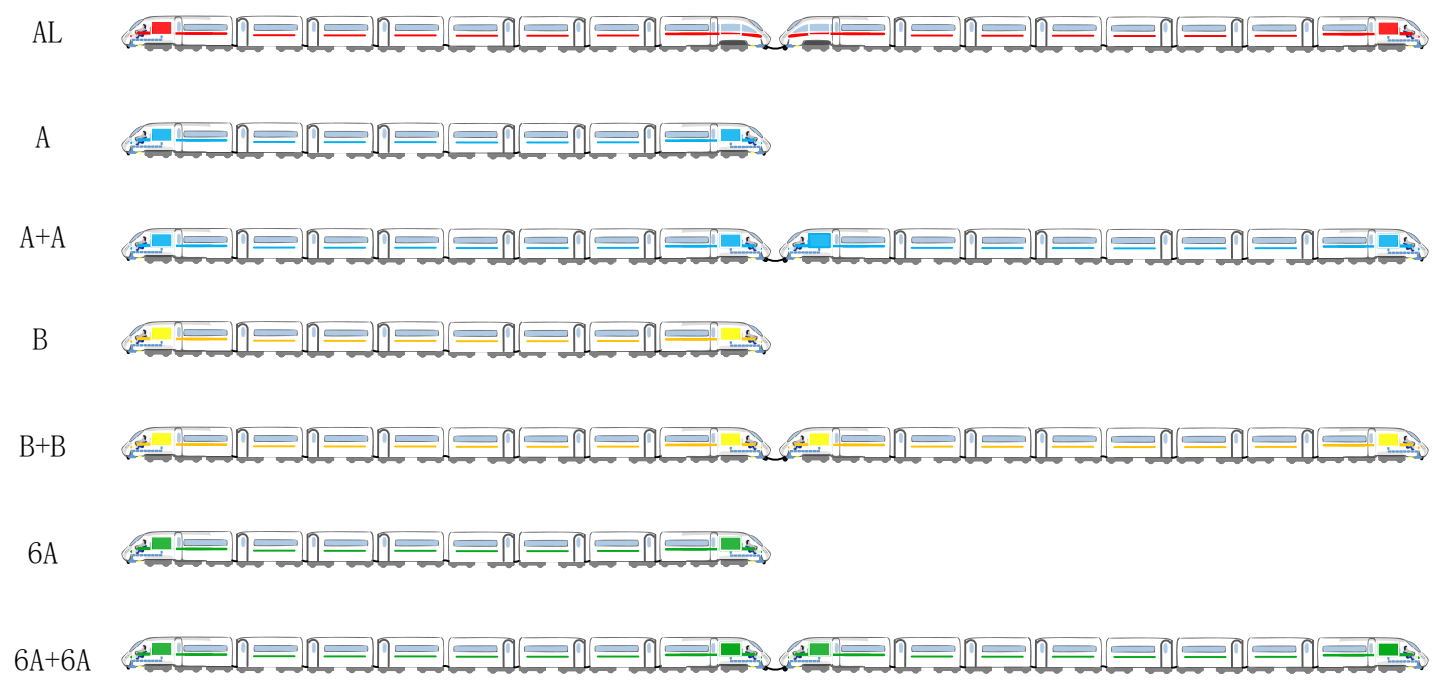

Fig. 1. Examples of train compositions

All stations in the CHSR network are built to the same specifications. This limits the number of feasible compositions. In particular, any composition must have at least 8 cars, but not more than 16 cars. This is equivalent to having one or two unit types in each composition since each unit contains exactly 8 cars. Coupling and decoupling can take place at the front or the rear of each unit.

Solving the rolling stock scheduling problem involves assigning a composition to each of the trips specified in the timetable. Composition changes are possible and may be beneficial for utilizing the available seat capacity better; however, only stations that are directly connected to depots can be used to facilitate a composition change. A depot is an area of tracks located close to a station and provides parking and maintenance services for idle units. Any (de)coupled unit must be shunted to (retrieved from) the depot. Coupling and decoupling units involve additional drivers and time and is therefore generally not preferred. If none of the delimiting stations of a trip has a depot, the assigned composition to each of the trips must be the same. Therefore, when solving the rolling stock scheduling problem, we work on an aggregated trip level, where 
some individual trips are aggregated together according to actual operational requirements. In the remainder of this paper, the term trip will refer to an aggregated trip. If two trips can be consecutively performed, this is referred to as a connection.

All rolling stock units are assumed to be initially placed in depots. Each unit will typically depart from a depot, perform a series of trips, and then return to a depot (not necessarily the same as the departure depot) at the end of the planning horizon. At the CHSR, overnighting is possible at stations that are located far from the depots (e.g., stations that located more than $2000 \mathrm{~km}$ from the depots). For those stations, there is insufficient time to deadhead an empty composition in order to begin a timetabled early morning trip. Deadheading simply refers to repositioning an empty composition. The deadheading movement is not specified in the timetable. We consider a two-day planning horizon as it is possible for all units to return to a depot within this time period. In order to ensure that there are enough rolling stock units available at each of the depots to carry out the timetable on the day immediately following a two-day planning horizon, it is important to balance the number of units available at each depot at the beginning and end of the planning horizon. Note that for this balance, the physical units need not be the same, but there should be the same number of units of each unit type. From a modelling perspective, we do allow a difference at the end of the planning horizon; however, an imbalance is penalized.

Adding maintenance requirements to the rolling stock scheduling problem creates a layer of complexity that can be difficult to incorporate into the existing state-of-the-art models for rolling stock scheduling without maintenance requirements. Depending on the maintenance requirements of an individual unit, certain sequences of trips might not be possible to perform. At the CHSR, preventive maintenance is routinely applied to all units. In particular, the need for maintenance of an individual unit depends on the running time of the unit and its accumulated mileage since its last maintenance check. Rolling stock units are subject to five levels of maintenance. In this paper, we focus on level one maintenance (or daily maintenance). This stipulates that all rolling stock units undergo a maintenance check every two days, or when the accumulated mileage exceeds $5,500 \mathrm{~km}$.

The rolling stock scheduling problem that we consider therefore involves not only determining the best composition for each trip, but also deciding how to assign compositions in such a way that the trip sequences assigned to each individual unit do not violate the maintenance requirements specified. For the CHSR safety, operational cost and efficiency are the three most important aspects of a rolling stock schedule. An efficient schedule is a schedule where the total deadhead mileage is minimized. We therefore seek a rolling stock schedule that satisfies all operational requirements, and which essentially minimizes a weighted combination of the operation cost, the total deadhead mileage and any penalties arising from rolling stock type imbalances.

We conclude this section with an example that visually illustrates the problem of rolling stock scheduling with maintenance requirements at the CHSR. Let us assume that there is a CHSR network with five main stations, one depot and 14 daily trips. This is depicted in Fig. 2. The stations appear on the vertical axis, where station $s_{2}$ is a depot station. Time appears on the horizontal axis and every trip is visualized as an arc between the two stations it connects. Each trip is numbered, and the minimal number of units needed for the trip is also stated. As an example, "1-II" refers to trip one and states that at least two rolling stock units are needed. We 
further assume that one day's operating time ranges from 6:00 to 24:00. All rolling stock units will depart from the depot not earlier than the start of the planning horizon and must return to the depot no later than the end of the planning horizon. Two types of rolling stock can be used on this network. Type AL is an older type that can have only one composition with two units (i.e., 16 cars). Type B is a newer type that can (de)couple at the depot station.

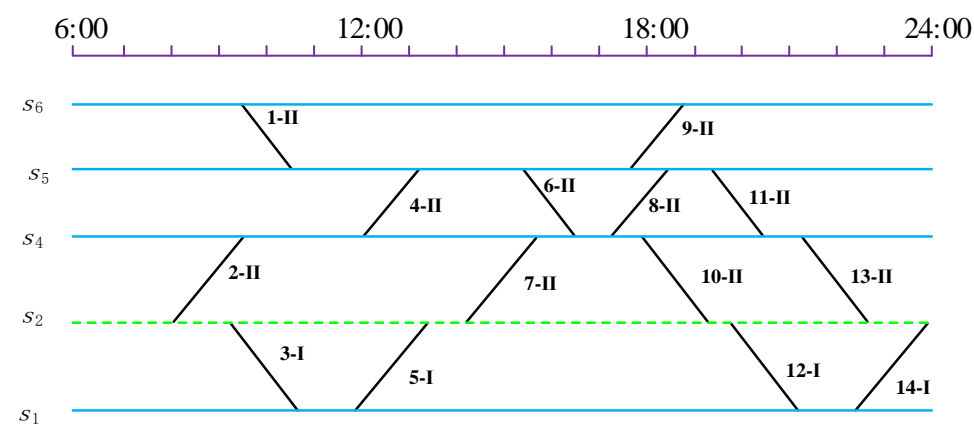

Fig. 2. Illustration of the timetable

Trip one does not have a previous trip. There is insufficient time to deadhead rolling stock units from the depot to serve this trip (the rolling stock units would otherwise have to depart before 6:00 am to get there due to the long deadheading distance). Consequently, this trip should be served on the second day (an overnight stop at the station is unavoidable). Figures 3 and 4 provide two feasible rolling stock schedules for this illustrative small network, showing possible trip sequences and compositions.

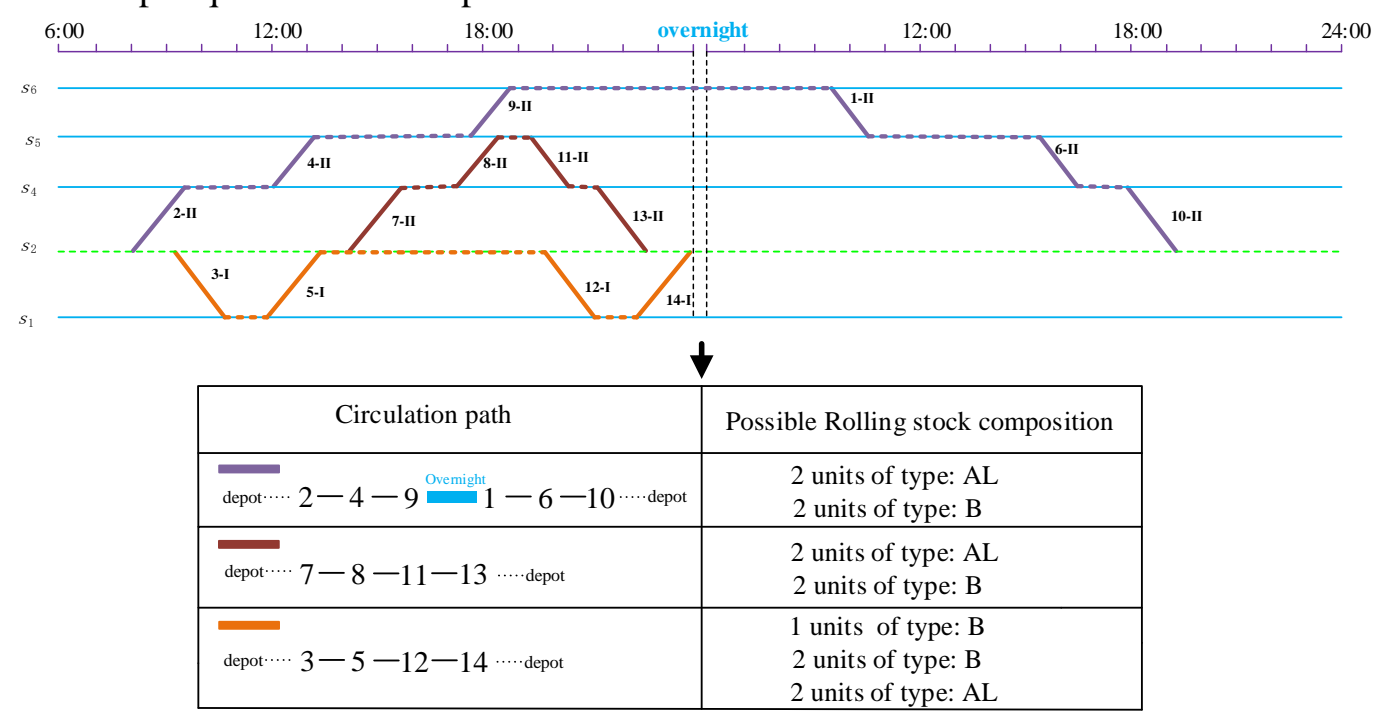

Fig. 3. Schedule 1 


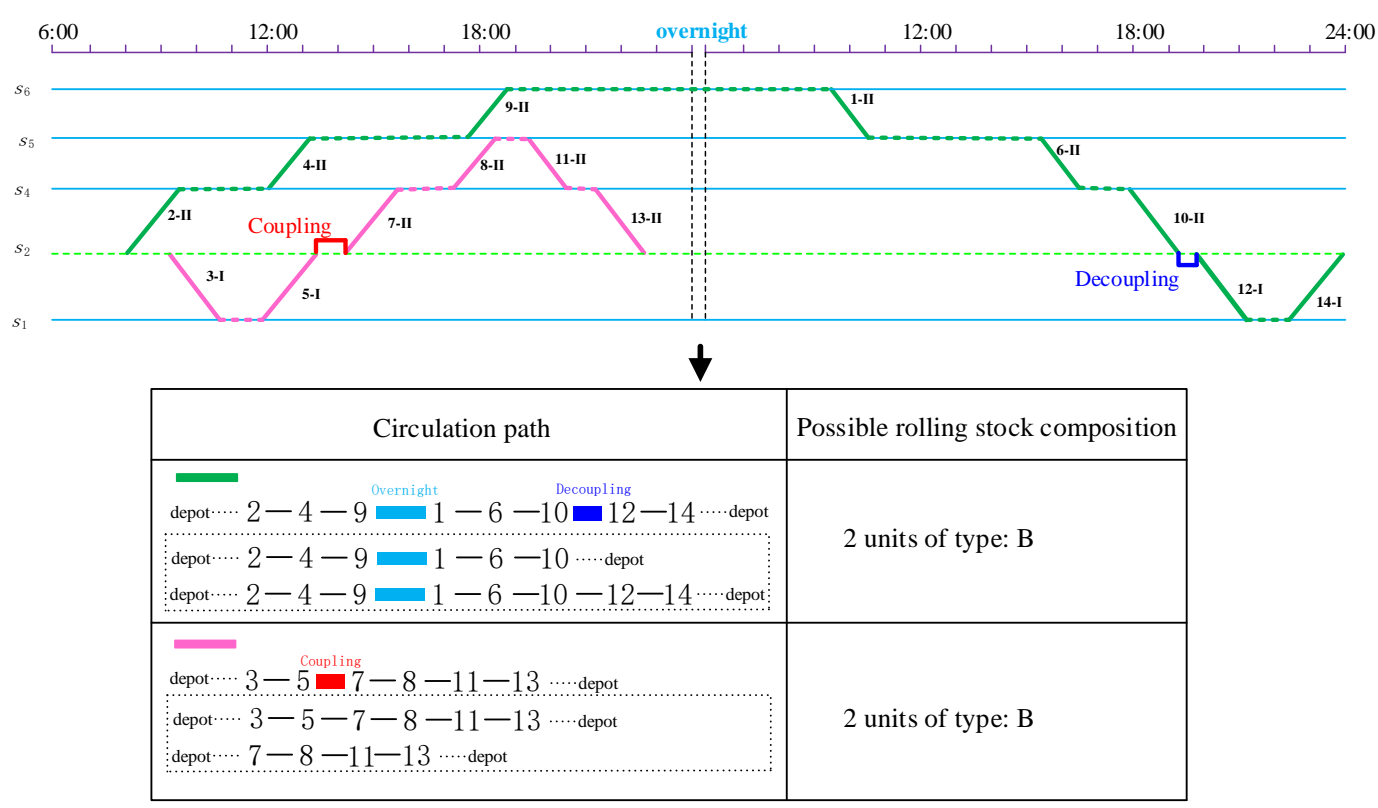

Fig. 4. Schedule 2

The first schedule contains no composition changes in the given trip sequences identified. As such, either type AL or type B units can be used. According to the composition rules at the CHSR, three different rolling stock compositions are possible for the third trip sequence. In the second schedule, however, both trip sequences incur composition changes. Only unit type B can be used in this schedule. Individual trip sequences are given within the dotted boxes and naturally converge (diverge) at the coupling (decoupling) location. This example considers a very small network; however, it nicely illustrates that there are a number of feasible rolling stock schedules. Deciding what composition should be assigned for each trip is not trivial, and this is complicated further by maintenance requirements. When considering maintenance, a trip sequence can only be assigned to a unit if the time and mileage consumption of the trip sequence is less than the remaining time and mileage of the unit. Obviously, there is no guarantee that the optimal schedule without maintenance is even feasible for the case where maintenance is considered; however, we believe it is a good place to start. Our proposed methodology exploits this reasoning.

In practice, it is very difficult for a dispatcher to foresee the implications of a decision. It is almost impossible to obtain a good rolling stock schedule in a short amount of time. Depending on the dispatcher, the same timetable may receive a different rolling stock schedule. Typically, manually produced rolling stock schedules will have some waste (e.g., unnecessarily increase operational cost or deadhead mileage) resulting in a lower unit efficiency. In this paper, we aim at determining an optimal rolling stock schedule that is also feasible with respect to maintenance requirements using a two-stage model. Automatically constructing such schedules will increase the efficiency of the units.

\section{Mathematical formulations}

In this section, we present the mathematical models and the algorithm for solving the rolling stock scheduling problem with maintenance requirements. We begin by emphasizing some assumptions made in this work.

- The timetable is known before scheduling any rolling stock units. This is a standard assumption and is consistent with what is done in practice (see e.g., Nielsen et al. (2012)). 
- Not all stations can be used for overnight parking. Stations that can be used have been approved by the CHSR.

- It is possible for deadheading units to connect trips. These "artificial" trips are added to the timetable and facilitate the empty repositioning of units. All deadheading possibilities have been generated in collaboration with, and approved by, the CHSR.

- All rolling stock units must go through a coupling and a decoupling procedure at the start and end of every trip sequence. In other words, even if the composition contains only one unit, some shunting procedures are necessary when departing from (or arriving at) the depot. This is also termed (de)coupling.

- Depots are assumed to have infinite capacity to accommodate all composition changes. This reflects that in the current system the smallest depot could accommodate all units if necessary. This is not a limiting assumption in our case as the depots are sufficiently big to hold all units if necessary.

Our approach is built around the idea of decomposing the problem into two distinct components, which are then solved separately. In the first stage, we solve the rolling stock scheduling problem ignoring all maintenance requirements. Efficient methodologies are available for solving this, and we make use of the arc-flow modeling ideas of Fioole et al. (2006) and Haahr et al. (2016). The arc-flow model of Haahr et al. (2016) is mostly based on Fioole et al. (2006), but has some slight changes in how the depot inventory is modelled. In order to be able to accurately implement the maintenance requirements, we introduce a time-based depot inventory variable to the model of Haahr et al. (2016), instead of having a depot inventory variable associated with composition changes. In addition, we also introduce two new variables related to composition changes to further help when generating all possible trip sequences. Finally, instead of finding just one solution to this problem, we determine up to a fixed number of $\mathrm{N}$ candidate rolling stock schedules. The optimal rolling stock schedule without maintenance can be used to benchmark the quality of the schedule when maintenance is included; however, there is no guarantee that the solution to this problem (or the second best, or third best for that matter) is feasible when incorporating maintenance. By determining the set of feasible candidate schedules and considering them in increasing order of cost, we do, however, have a structured way of assessing maintenance feasibility. The second stage involves iteratively considering sets of $F$ best rolling stock schedules from the first stage. In other words, in the second stage, $F$ MILP problems are solved where each one assesses the feasibility of the corresponding rolling stock schedule from a maintenance perspective. The MILP model assigns individual units to an enumerated list of trip sequences from the rolling stock schedule. We now discuss the proposed mathematical models.

\subsection{Stage 1}

The first stage is based on the arc-flow model. To identify which compositions to assign to which trips, we use a variation of the arc-flow model proposed in Haahr et al. (2016). The notation used in the arc-flow model is introduced in Tables 1 and 2.

\section{TABLE 1 Definition of Sets, Indices, and Parameters}

\begin{tabular}{ll}
\hline Notation & Description \\
\hline$S$ & Set of stations \\
$D$ & Set of depots \\
$P$ & Set of rolling stock unit types \\
$C$ & Set of all compositions
\end{tabular}




\begin{tabular}{|c|c|}
\hline$m e T$ & Set of timetabled trips \\
\hline deadheadT & Set of deadheading trips \\
\hline sourceT & $\begin{array}{l}\text { Set of deadheading trips that connect depot and corresponding depot station, } \\
\text { source } T \subset \text { deadheadT }\end{array}$ \\
\hline overT & Set of all overnight trips \\
\hline$T$ & Set of all trips, $T=$ deadhead $T \bigcup m e T \bigcup$ over $T$ \\
\hline Cnn & Set of all connections \\
\hline$s_{r}$ & The first trip of connection $r \in C n n$ \\
\hline$t_{r}$ & The second trip of connection $r \in C n n$ \\
\hline$g_{r}$ & The station at which connection $r \in C n n$ take place \\
\hline$C_{\mathrm{c}, \mathrm{c}^{\prime}}^{r}$ & $\begin{array}{l}\text { Set of allowable composition changes for connection } r \text {. This set contains } \\
\text { composition } c \text { and composition } c^{\prime} \text { are allowed for first trip } s_{r} \text { and second } \\
\text { trip } t_{r} \text { respectively }\end{array}$ \\
\hline $\operatorname{bod}_{d}^{p}$ & $\begin{array}{l}\text { Inventory of units of type } p \in P \text { at depot } d \in D \text { at the beginning of the } \\
\text { planning horizon }\end{array}$ \\
\hline $\operatorname{eod}_{d}^{p}$ & $\begin{array}{l}\text { The target inventory of units of type } p \in P \text { at depot } d \in D \text { at the end of the } \\
\text { planning horizon }\end{array}$ \\
\hline shuntCost $t_{c, c^{\prime}}^{r}$ & $\begin{array}{l}\text { The cost incurred when changing from composition } c \text { to composition } c^{\prime} \text { on } \\
\text { connection } r\end{array}$ \\
\hline operCost $t_{c}^{t}$ & The operational cost of using composition $c \in C$ on trip $t \in T$ \\
\hline impenEod & The penalty for a planning horizon storage imbalance \\
\hline $\operatorname{couple}_{c, c^{\prime}}^{p}$ & $\begin{array}{l}\text { The number of units of type } p \in P \text { that are coupled when changing from } \\
\text { composition } c \text { to composition } c^{\prime}\end{array}$ \\
\hline $\operatorname{decouple}_{c, c^{\prime}}^{p}$ & $\begin{array}{l}\text { The number of unit type } p \in P \text { that are decoupled when changing from } \\
\text { composition } c \text { to composition } c^{\prime}\end{array}$ \\
\hline tDecoupling $_{r}$ & $\begin{array}{l}\text { Decoupling time necessary on connection } r \text {. This is the amount of time that } \\
\text { must elapse before a decoupled unit can be used again. }\end{array}$ \\
\hline tCoupling $_{r}$ & $\begin{array}{l}\text { Coupling time necessary on connection } r \text {. The amount of time necessary to } \\
\text { perform a coupling }\end{array}$ \\
\hline Time & The planning horizon in minutes \\
\hline
\end{tabular}

TABLE 2 List of variables

\begin{tabular}{|c|c|}
\hline Variable & Description \\
\hline$y_{t}^{c}$ & $\begin{array}{l}\text { Binary decision variable indicating whether or not composition } c \in C \text { is used } \\
\text { on trip } t \in T\end{array}$ \\
\hline$i_{d}^{p}$ & $\begin{array}{l}\text { Non-negative decision variable stating the imbalance of unit type } p \in P \text { at } \\
\text { depot } d \in D\end{array}$ \\
\hline$X_{c, c^{\prime}}^{r}$ & $\begin{array}{l}\text { Binary decision variable deciding whether composition } c \in C \text { and } c^{\prime} \in C \text { are } \\
\text { used for trip } s_{r} \text { and } t_{r} \text { respectively }\end{array}$ \\
\hline$v 1_{r}^{p}, v 2_{r}^{p}$ & $\begin{array}{l}\text { Non-negative integer decision variables which represent the number of unit } \\
\text { type } p \in P \text { coupled and decoupled in the connections } r \in C n n\end{array}$ \\
\hline$h_{r, p}^{d}, g_{r, p}^{d}$ & $\begin{array}{l}\text { Binary decision variables deciding whether the coupled and decouple unit } \\
\text { type } p \in P \text { in the connection } r \in C n n \text { is from depot } d \in D \text { respectively }\end{array}$ \\
\hline $\operatorname{storage}_{p, d}^{\text {time }}$ & $\begin{array}{l}\text { Non-negative decision variable stating the inventory of type } p \in P \text { at depot } \\
d \in D \text { at minute time time } \in \text { Time }\end{array}$ \\
\hline deadheadMile & The total deadhead mileage during one planning horizon \\
\hline overTime & The total overnighting time of the schedule \\
\hline
\end{tabular}

At the CHSR, the focus is primarily on profit and efficiency. In order to make more profit, 
the combined operational cost and shunting cost should be minimized. Efficiency is defined as the effective running mileage during the planning horizon. To maximize this, deadhead mileage should be minimized. Furthermore, unnecessary overnighting and depot imbalances should be avoided. The final objective function therefore consists of five parts: the combined operational cost, the shunting cost, depot imbalance penalties, total deadhead mileage, and the total overnight time. This is formally stated in Eq. (1):

$$
\begin{aligned}
\min z= & \sum_{t \in T} \sum_{c \in C} \operatorname{operCost}_{c}^{t} \cdot y_{c}^{t}+\sum_{r \in C n n} \sum_{c \in C} \sum_{c^{\prime} \in C} \operatorname{shuntCost}_{c, c^{\prime}}^{r} \bullet X_{c, c^{\prime}}^{r} \\
& +\sum_{p \in P} \sum_{d \in D} \text { impenEod }_{\bullet} \boldsymbol{i}_{d}^{p}+\alpha \bullet \text { overTime }+\beta \bullet \text { deadheadMile }
\end{aligned}
$$

In the objective function, $\alpha$ and $\beta$ represent the penalty parameters. The variable overTime represents the total overnight time of the schedule. This can be computed as follows:

$$
\text { overTime }=\sum_{\substack{r \in C n n \\ s_{r} \text { OoverT }}} \sum_{c \in C} \sum_{c^{\prime} \in C} X_{c, c^{\prime}}^{r} \text { overtime }_{s_{r}}
$$

where the overtime $_{t}$ means the overnight time of trip $t$. The running distance of all deadhead trips used in the solution can be calculated in a similar way:

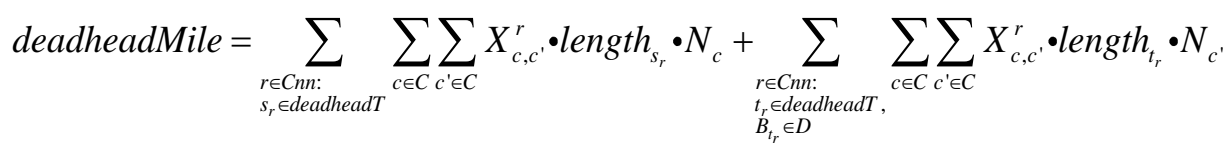

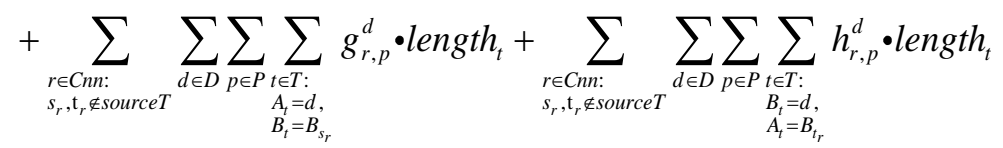

where the length $h_{t}$ means the length of trip $t, N_{c}$ represents the number of rolling stock units in composition $c$, and $A_{t}$ and $B_{t}$ refer to the arrival and departure station (depot) of trip $t$ respectively.

The flow model is subject to a number of constraints. We now introduce each of these in turn. To obtain a feasible rolling stock schedule, each timetabled trip must be operated by one and only one composition. As the timetable is repeated daily in a two-day planning horizon, each trip is replicated twice, once on the first day and once on the second day. The set of trips on the first day is denoted oneT while the set of trips on the second day is given by twoT . Naturally, meT $=$ one $T \bigcup t w o T$. The departure time of the second trip is exactly 1440 minutes after the departure of the first trip. As such, exactly one of the two trips must receive a valid composition. This is expressed as follows:

$$
\sum_{c \in C} y_{t}^{c}+\sum_{c \in C} y_{t^{\prime}}^{c}=1 \quad \forall t \in \text { one } T, t^{\prime} \in \text { two } T
$$

Deadheading possibilities are not necessarily duplicated, but still must adhere to this uniqueness. For deadheading trips which do not link to the depots and overnight trips, constraint (4) reduces to

$$
\sum_{c \in C} y_{t}^{c} \leq 1 \quad \forall t \in \text { deadheadT Uover } T: \mathrm{A}_{t}, \mathrm{~B}_{t} \notin D
$$

Where $A_{t}$ and $B_{t}$ refer to the arrival and departure station (depot) of trip $t$ as mentioned before.

To ensure that feasible and consistent rolling stock compositions are assigned to the trips, constraints are needed to monitor the composition transitions between consecutive trips. Based on the number of units required for a given trip, there is a set of allowable compositions that 
the trip can receive. Each trip must receive one of its allowed compositions. These are stated as:

$$
\begin{aligned}
& \sum_{\substack{r \in \in n n: \\
s_{r}=t}} \sum_{s_{r}:\left(c, c^{\prime}\right) \in \mathrm{C}_{c, c^{\prime}}^{r}} X_{c, c^{\prime}}^{r}=y_{t}^{c} \quad \forall t \in T: A_{t}, \mathrm{~B}_{t} \notin \mathrm{D}, c \in C \\
& \sum_{\substack{r \in C n n: c^{\prime}:\left(c^{\prime}, c\right) \in C_{c}^{r}, c \\
t_{r}=t}} X_{c^{\prime}, c}^{r}=y_{t}^{c} \quad \forall t \in T: A_{t}, \mathrm{~B}_{t} \notin \mathrm{D}, c \in C
\end{aligned}
$$

The inventory of rolling stock types at every depot must remain non-negative throughout the planning horizon. In order to monitor the inventory levels precisely, the number of rolling stock units of type $p \in P$ that are (de)coupled to the composition at connection $r \in C n n$ must be determined. The following two constraints achieve this:

$$
\begin{aligned}
& v 1_{r}^{p}=\sum_{c \in C} \sum_{c^{\prime}:\left(c, c^{\prime}\right) \in C_{c, c^{\prime}}^{r}} X_{c, c^{\prime}}^{r} \bullet \text { couple } e_{c, c^{\prime}}^{p} \quad \forall r \in \text { Cnn }, p \in P \\
& v 2_{r}^{p}=\sum_{c \in C} \sum_{c^{\prime}:\left(c, c^{\prime}\right) \in C_{c, c^{\prime}}^{r}} X_{c, c^{\prime}}^{r} \bullet \operatorname{decoupl} e_{c, c^{\prime}}^{p} \quad \forall r \in C n n, p \in P
\end{aligned}
$$

The rolling stock inventory of type $p \in P$ at time $\in$ Time is equal to the initial inventory of the depot at the beginning of the planning horizon minus all units being coupled to compositions before time $\in$ Time plus all units which are available again after being decoupled from compositions in earlier connections. This can be expressed as:

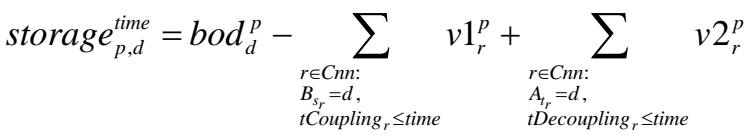

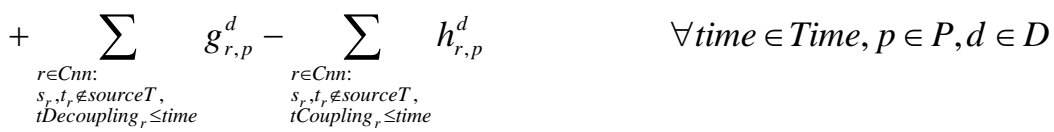

In Constraint (10), the used rolling stock units are divided into two parts: units that are (de)coupled at the depot and those that are (de)coupled at stations. The following constraints (11)-(13) are for the rolling stock units (de)coupled at stations. These are not necessary for the model, but help us to identify the corresponding depot of (de)coupled units.

$$
\begin{aligned}
& \sum_{d \in D} g_{r, p}^{d}=v 2_{r}^{p} \quad \forall r \in \text { Cnn }: s_{r}, t_{r} \notin \text { source } T ; p \in P \\
& \sum_{d \in D} h_{r, p}^{d}=v 1_{r}^{p} \quad \forall r \in \text { Cnn }: s_{r}, t_{r} \notin \text { source } T ; p \in P
\end{aligned}
$$

Finally, the imbalance of units of type $p \in P$ at depot $d \in D$ can be calculated as:

$$
i_{d}^{p} \geq\left(\operatorname{bod}_{d}^{p}-\operatorname{eod}_{d}^{p}\right)-\text { storage }_{p, d}^{\text {etime }}+\text { storage }_{p, d}^{\text {btime }} \quad \forall d \in D, p \in P
$$

where etime and btime indicate the start and end times of the planning horizon respectively. The mathematical model defined by (1) to (13), along with the variable domains specified by Table 2, will provide a feasible rolling stock schedule in the absence of maintenance requirements.

\subsection{Stage 2}

To determine whether a rolling stock schedule that is feasible for the arc-flow model is also feasible with respect to individual unit maintenance restrictions, an MILP that assigns individual trip sequences to the units can be defined. This model is termed the Trip Sequence Assignment Model (TSAM). The input to this model is an enumerated list of trip sequences from a solution to the arc-flow model. More specifically, given the solution from the arc flow 
model, one can enumerate all possible trip sequences for each of the rolling stock units. The notations and decision variables used in the TSAM are introduced in Tables 3 and 4.

TABLE 3 Definition of Sets, Indices, and Parameters

\begin{tabular}{ll}
\hline Notation & Description \\
\hline Rolling & Set of all rolling stock units, roll $\in$ Rolling \\
$L$ & Set of all possible individual trip sequences \\
$L_{\text {roll }}$ & $\begin{array}{l}\text { Set of possible individual trip sequences for a single rolling stock unit } \\
\text { roll } \in \text { Rolling } \text { with considering maintenance requirements }\end{array}$ \\
assCost $t_{\text {roll }}$ & $\begin{array}{l}\text { Integer parameter representing the artificial cost of a single rolling stock unit } \\
\text { roll } \in \text { Rolling }\end{array}$ \\
& Binary parameter indicating whether or not trip $t \in T$ is contained in trip \\
$a_{l}^{t}$ & sequence $l \in L_{\text {roll }}$ \\
$b_{t}$ & Integer parameter representing the number of units needed by trip $t \in T$. Note \\
& that this parameter is decided by the results of $y_{t}^{c}$ in the arc-flow model \\
\hline
\end{tabular}

TABLE 4 List of variables

\begin{tabular}{ll}
\hline Variable & Description \\
\hline$j_{\text {roll }}^{l}$ & $\begin{array}{l}\text { Binary decision variable deciding whether rolling stock roll } \in \text { Rolling is used } \\
\text { on individual trip sequences } l \in L_{\text {roll }} .\end{array}$ \\
\hline
\end{tabular}

If multiple feasible solutions exist for the TSAM, we are interested in the one that use the least number of rolling stock units. Thus, we introduce an artificial activation cost for each rolling stock unit. Reducing the number of used rolling stock units can also reduce the maintenance times. Activating a recently maintained unit is seen to be an expensive option and therefore incurs a higher cost. Our objective is therefore:

$$
\min z=\sum_{l \in L} \sum_{\text {roll } \in \text { Rolling }} j_{\text {roll }}^{l} \bullet \text { ass Cost } t_{\text {roll }}
$$

There are two main types of constraints in the MILP model. The first set of constraints ensures that the number of rolling stock units assigned to each trip is consistent with the solution to the arc-flow model. The second set of constraints specifies that each unit receives at most one trip sequence. These are formulated as constraints (15) and (16) respectively.

$$
\begin{aligned}
& \sum_{\text {roll } \in \text { Rolling }} \sum_{l \in L_{\text {rol }}} a_{l}^{t} \cdot \dot{j}_{\text {roll }}^{l}=b_{t} \quad \forall t \in m e T \\
& \sum_{l \in L_{\text {roll }}} j_{\text {roll }}^{l} \leq 1 \quad \forall \text { roll } \in \text { Rolling }
\end{aligned}
$$

For a given unit roll $\in$ Rolling, the set $L_{\text {roll }}$ can be determined from the solution to the arc-flow model. Using the anonymous unit type flow values from the arc-flow model, and given the unit's type, it is possible to enumerate all trip sequences that start at the unit's initial depot and terminate at another depot. For each such trip sequence, its distance and running time are known. Therefore it is easy to identify which trip sequences are not possible for the unit given the unit's remaining mileage and time. These are removed from consideration, and $L_{\text {roll }}$ contains only maintenance feasible trip sequences for the unit roll. That is, during a planning horizon, a trip sequence $l$ in $L_{\text {roll }}$ should satisify the time and distance restricitons in constraints (17)-(18): 


$$
\begin{aligned}
& \sum_{t} a_{l}^{t} \cdot \text { length }_{t}+\text { Slength }_{\text {roll }} \leq 5500 \quad \begin{array}{l}
\forall \text { roll } \in \text { Rolling }, l \in L_{\text {roll }}: \\
\text { Type }_{\text {roll }}=l_{\text {type }} \cap l_{\text {depot }}=\text { Depot }_{\text {roll }}
\end{array} \\
& \begin{array}{c}
\quad \forall \text { roll } \in{\text { Rolling }, l \in L_{\text {roll }}:}_{l_{\text {time }}+\text { Stime }_{\text {roll }} \leq 2880} \text { Type }_{\text {roll }}=l_{\text {type }} \cap l_{\text {depot }}=\text { Depot }_{\text {roll }}
\end{array}
\end{aligned}
$$

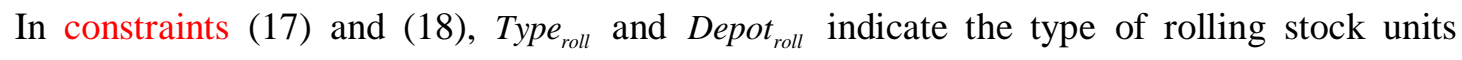
roll $\in$ Rolling and the initial parking depot of roll $\in$ Rolling $. l_{\text {type }}, l_{\text {depot }}$ and $l_{\text {time }}$ represent the required type of rolling stock unit, required departure depot and the running time of trip sequence $l$, respectively.

If a feasible solution to the TSAM is found, a maintenance feasible rolling stock schedule has been found. It could be necessary to consider several TSAM problems as there is no guarantee that the first one be optimal. In what follows, we provide an overview of the full algorithm.

\subsection{Algorithm}

Figure 5 provides a flow diagram of the proposed solution approach. We begin by solving the arc-flow model and populate a pool of up to $\mathrm{N}$ rolling stock schedules. We then iteratively solve F TSAM problems to assess maintenance feasibility of each of the rolling stock schedules. The algorithm stops when an iteration of the TSAM problems produces at least one feasible solution. In the case that it provides more than one, we keep the one with the lowest objective value. Here we can see that the first stage decides the operating costs of the schedule, and the corresponding second stage is used to check whether an assignment of rolling stock units that satisfies all the maintenance requirements can be obtained. If the algorithm exhausts all $\mathrm{N}$ solutions without finding a maintenance feasible solutions we go back to stage 1 to find solutions to the arc-flow model and take another loop.

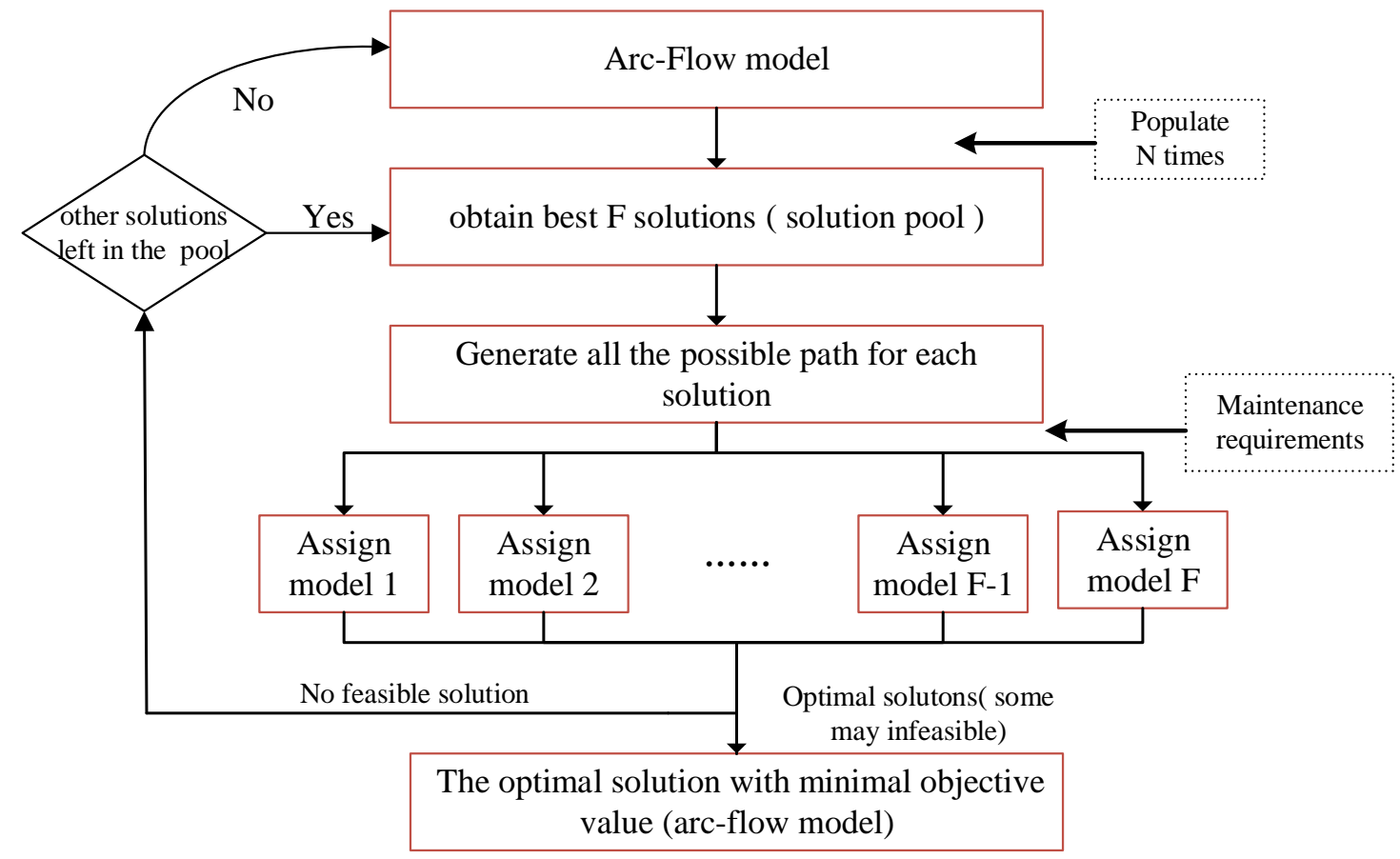

Fig. 5. Flow diagram for the proposed algorithm

The arc-flow model is programmed in C\#, and we use the commercial solver CPLEX to solve the resulting MILP problem. The optimal solution to this model can usually be found in a very short time (seconds). When solving the TSAM problem, it is important to consider all 
feasible trip sequences from the corresponding solution to the arc-flow model. Enumerating trip sequences could become problematic for large datasets with many possible rolling stock compositions. For the CHSR, however, there are a small number of compositions and few locations where compositions changes can occur. Enumeration is therefore not a problem. From the decision variables $X_{c, c^{\prime}}^{r}$ of the arc-flow model, the selected connections and associated trip compositions can be identified. To obtain all trip sequences for a solution to the arc-flow model we construct a graph in which each node represents a trip and each edge identifies a chosen connection. Identifying all feasible trip sequences amounts to finding all paths from any starting node to any terminating node in this graph. Here a small example with 34 trips is provided. Considering a solution to the arc-flow model in which 17 connections are chosen, the corresponding directed graph is shown in Fig. 6.
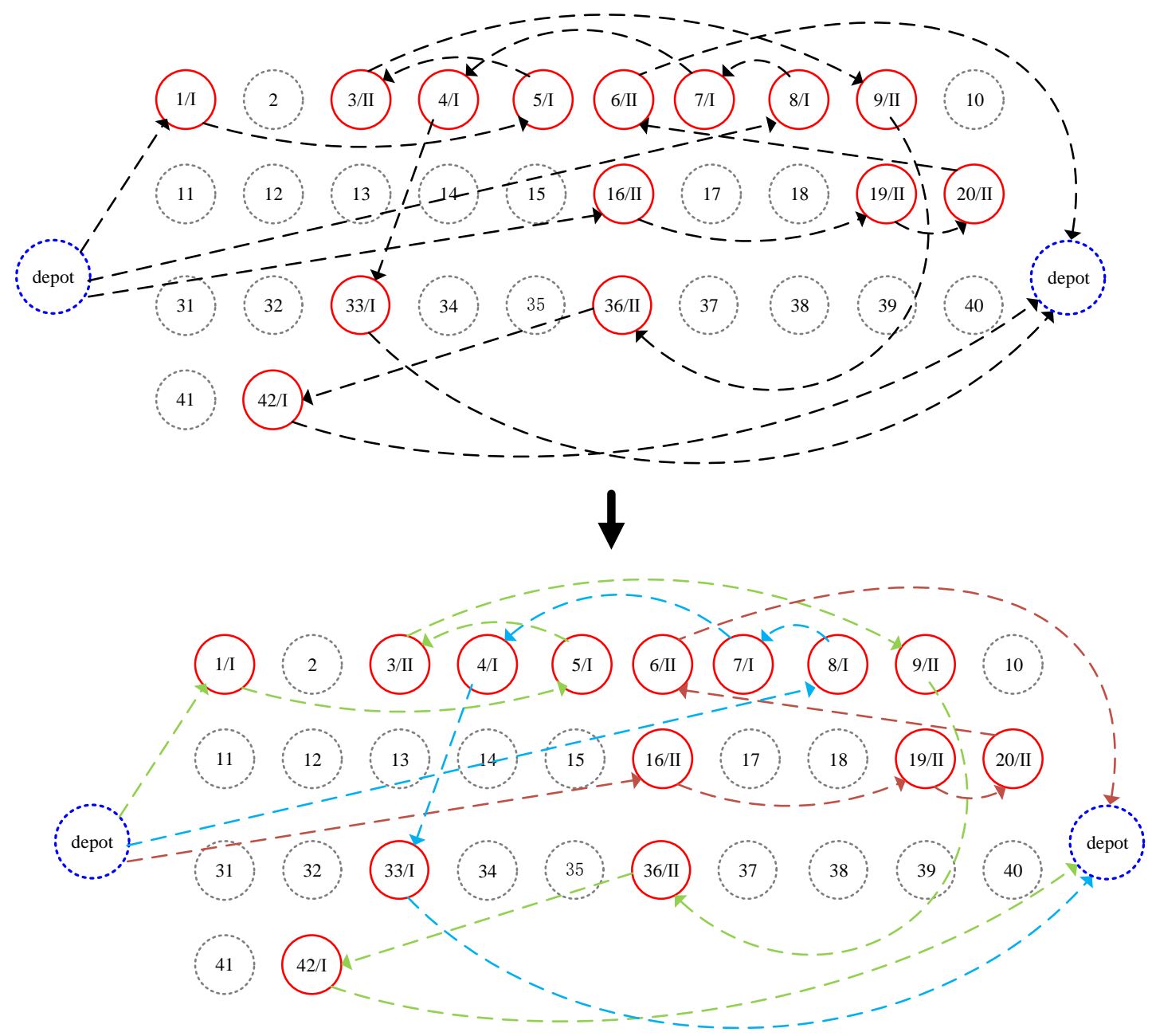

Fig. 6. Directed graph based on one arc-flow solution

Blue nodes indicate depots, while red nodes are associated with chosen trips. The numbers within a red circle indicate the trip number and the number of rolling stock units needed for the trip. Trips that are not selected are indicated with gray nodes. Information regarding the rolling stock type is also known, but is not displayed in the figure. 
The following steps summarize how all trip sequences for a single rolling stock unit can be determined.

Step 1: Based on the results of the arc-flow model, identify all unit flows (from depot to depot), as shown in Fig. 6. Divide the flows into two sets: one set contains flow without composition changes outside the depot, while the other contains composition changes outside the depot.

Step 2: For the flows without composition changes outside the depot, split each flow into individual trip sequences according to the parameter $y_{t}^{c}$ from the arc-flow model. Except for the number of rolling stock units, other features remain unchanged. An example of this is provided in Fig.7.
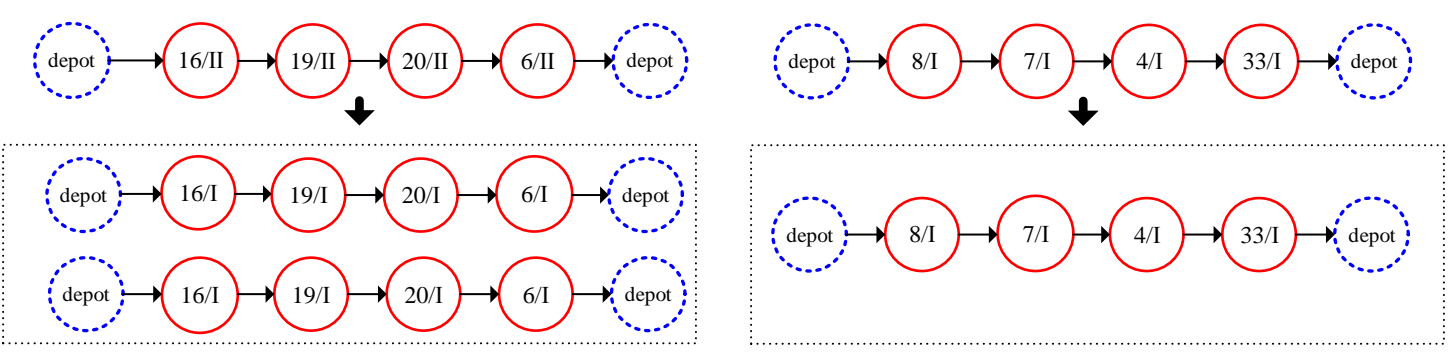

Fig. 7. Illustration of flow without a composition change

Step 3: For the flows with composition changes outside the depot, parameters $h_{r, p}^{d}, g_{r, p}^{d}$ of the arc-flow model highlight the connections that have a composition change (i.e., units are coupled or decoupled). Furthermore, the associated depots are also known. Now, it is easy to add a new edge (from corresponding depot to the station where composition changes happened) to the respective connection to split the flow into a single rolling stock unit flow. Then, it is possible to generate all possible trip sequences in the resulting graph by using a depth first search method. An example of this is shown in Fig. 8.

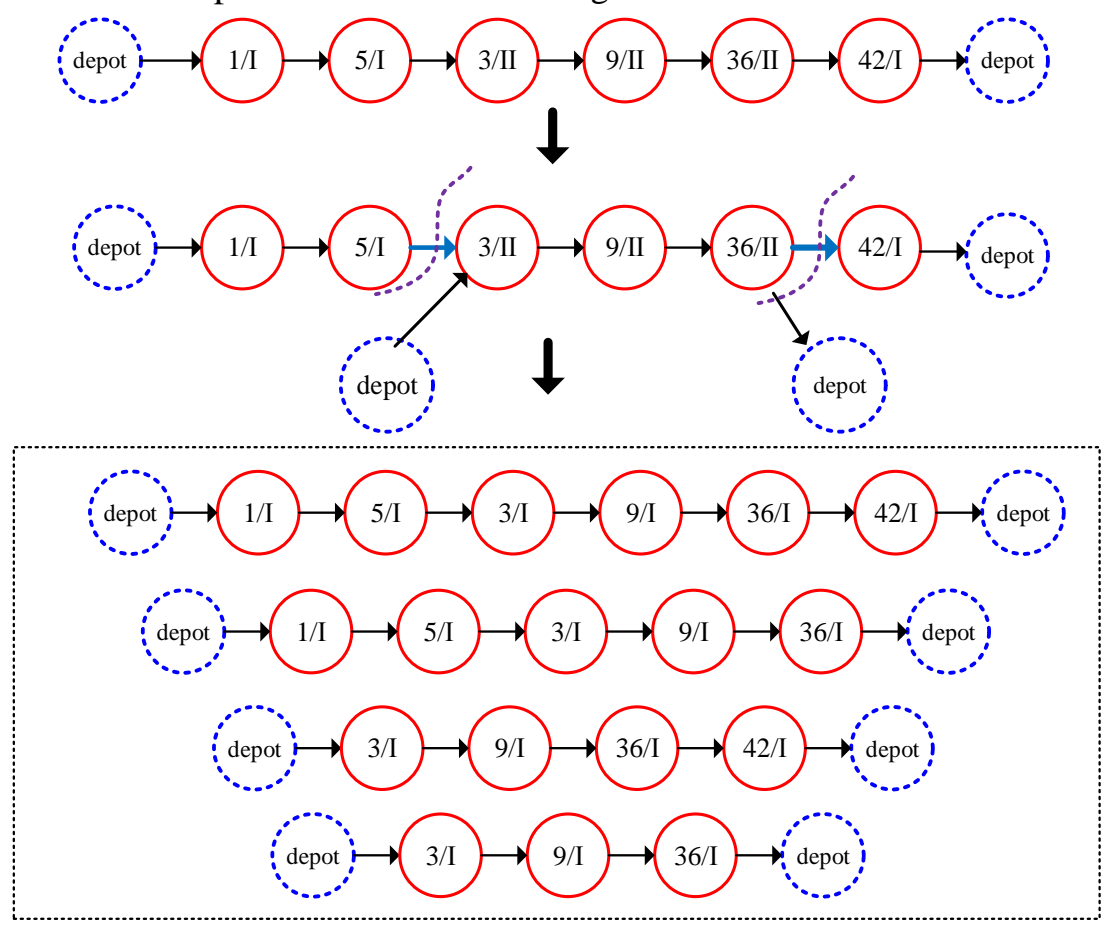

Fig. 8. Illustration of flow with a composition change 
Step 4: After steps 2 and 3, the arc-flow model solution has essentially been represented as partial trip sequences for a single rolling stock unit. Note that a rolling stock unit may take several partial trip sequences as long as these can connect to each other. Two partial sequences can be connected if they are both for the same unit type, the terminating depot of the first sequence is the same as the originating depot of the second sequence, and the depot arrival time of the first sequence is earlier than the depot departure time of the second sequence. Based on the partial trip sequences, it is easy to enumerate all full trip sequences.

\section{Computation results}

In this section, we test and compare the performance of the proposed methodology on actual instances from the CHSR. In particular, we look at the rail network of China Zhengzhou Railway Company. Section 5.1 introduces the relevant data, while Section 5.2 discusses the main results of our research. In Section 5.3 we consider the scalability of the proposed approach.

\subsection{Parameters and settings}

An overview of this network is provided in Fig. 9. The Zhengzhou Railway is one of the most important hubs in the CHSR network. The world's longest HSR line (Beijing to Guangzhou high-speed railway) is included in this network. The length of this line is about 2,298 km. The network comprises 36 main stations and two depots, and both depots can be reached from the stations of Zhengzhou Dong and Zhengzhou. Two different timetables are obtained from the Zhengzhou Railway Company to test the proposed method. One is the timetable from 2017 (Data-1), and the other one is the current timetable (Data-2).

Prior to August 2018, there were three different rolling stock types: CRH380AL, CRH380A, and CRH380B. A new type of rolling stock, CRH6A, was added to this network after the timetable adjustment in August. There are a total of 91 units in Data-1 and a total of 98 units in Data-2. An overview of the unit type distribution by depot for each dataset is given in Table 5. The "Depot" column indicates the two available depots, i.e., Zhengzhoudong (ZZD) and Zhengzhou (ZZ) depots.

TABLE 5. Initial location of unit types

\begin{tabular}{cccccc}
\hline Data & Depot & CRH380AL & CRH380A & CRH380B & CRH6A \\
\hline \multirow{2}{*}{ Data-1 } & ZZ & 0 & 7 & 11 & - \\
& ZZD & 36 & 9 & 28 & - \\
\hline \multirow{2}{*}{ Data-2 } & ZZ & - & - & - & 8 \\
& ZZD & 34 & 16 & 40 & - \\
\hline
\end{tabular}

Each unit is assumed to have a known accumulated mileage and running time since its last maintenance check. According to the CHSR, on average, 47 units are maintained every day. The number of units that have just received their level one maintenance check is 47 for Data- 1 and 48 for Data-2. 


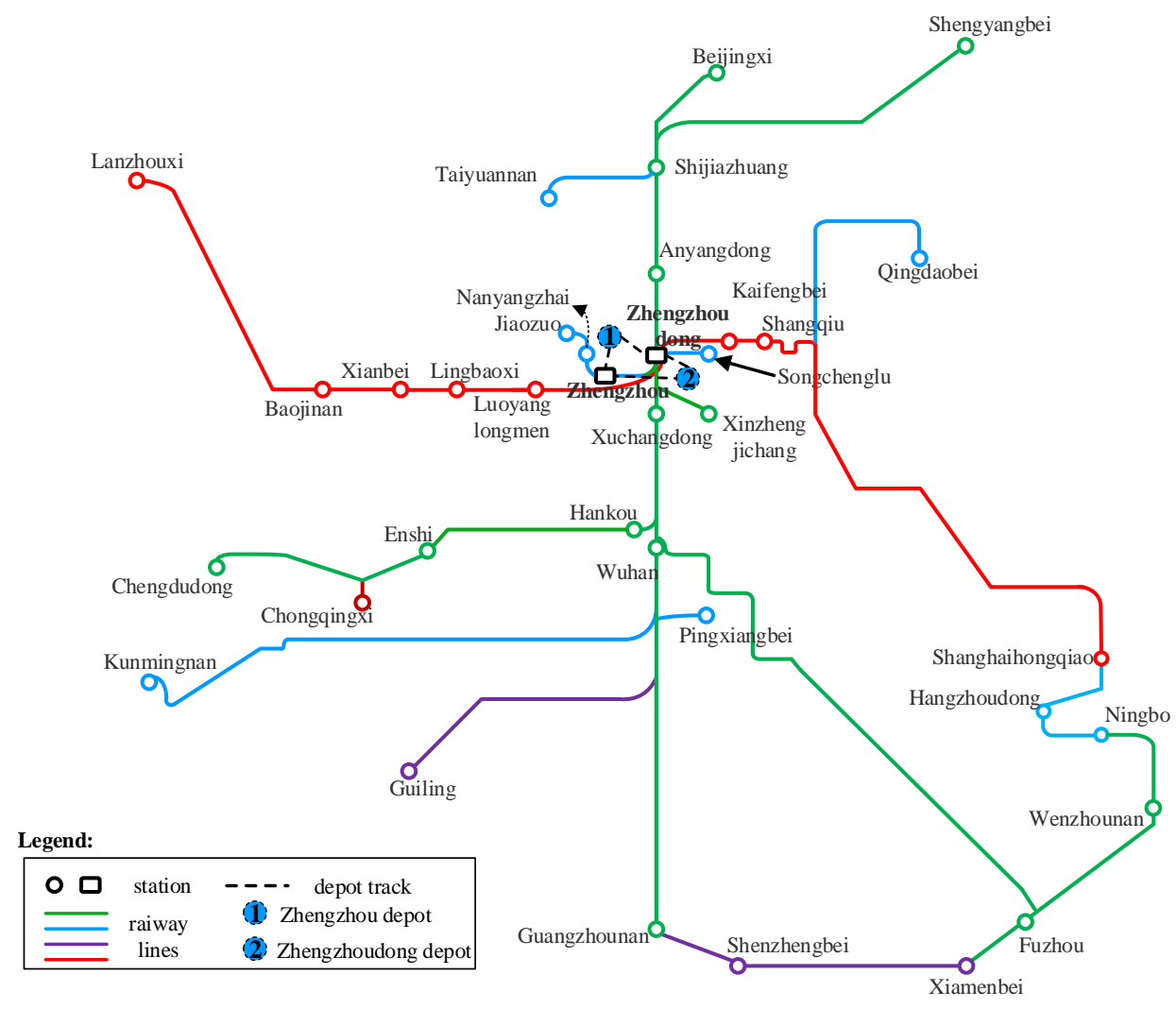

Fig. 9. Schematic map of the China's Zhengzhou Railway

As mentioned before, regardless of the type, all units contain 8 cars. The CRH380AL type is an older type and can only be used in one composition of 16 cars (two units). Rolling stock types CRH380A, CRH380B and CRH6A can be (de)coupled. Seven different possible compositions are listed in Table 6.

Table 6. The compositions of rolling stock units

\begin{tabular}{ccc}
\hline Composition name & Rolling stock type & Number of units \\
\hline CRH380AL-long & CRH380AL & 2 \\
CRH380A-long & CRH380A & 2 \\
CRH380A-short & CRH380A & 1 \\
CRH380B-long & CRH380B & 2 \\
CRH380B-short & CRH380B & 1 \\
CRH6A-long & CRH6A & 2 \\
CRH6A-short & CRH6A & 1 \\
\hline
\end{tabular}

The timetable for Data-1 was in operation between August 2017 and July 2018 and has 159 trips in total. When considering a two-day planning horizon, and after including some additional deadheading and overnight trips, there are 377 trips in total. Data-2 has 212 trips and a total of 490 trips in one planning horizon. Though the discussion with dispatchers, the penalty of deadhead mileage and overnight time are set to 1 and 4, respectively. For the imbalance penalty, we have set this to 100,000 to be consistent with previous work in this area (see e.g., Haahr et al. 2016).

The proposed methodology has been programmed in $\mathrm{C \#}$, in which the commercial solver CPLEX 12.8.0 is called to populate the arc-flow model and solve the resulting TSAMs. After 
carrying our preliminary tests, we set the CPLEX's solution pool intensity parameter to 3, the maximum number of MIP solutions generated for the solution pool during each call $(\mathrm{N})$ to the populate procedure is set to 1000 , and the number of best schedules chosen from the first stage (F) is 50. Other than this, default parameter values are used. For the stage 1, we allow any feasible solution to be added to the pool. A PC equipped with an Intel Core i7-4700MQ 2.40GHz CPU, 8 GB RAM, and a Windows 10-64 bit operating system is used for running all tests.

\subsection{Results}

We perform several computational tests using the provided datasets. In order to show the quality of solutions found by our algorithm, we consider the case with maintenance (M) and the case without maintenance (NM) for both datasets. With the rules of case NM, every solution found by the first stage is feasible in the second stage. Thus, the result of case NM gives us a lower bound of this problem. As mentioned before, the coupling and decoupling processes are allowed at specific stations in our model. However, in practice, there are usually no composition changes taking place outside of the depots. The reason for this is that it is easier for the dispatchers to manually schedule the rolling stock units. As a comparison, we also test the algorithm's performance when it is restricted to the same simplified planning rules used by the dispatchers (M-D). When restricted to the dispatcher's rules, the arc-flow model is modified to forbid composition changes outside of the depots, and in the TSAM, all units must return to the depots they originate from.

In practice, it is important to obtain rolling stock schedules for multiple planning horizons. Dispatchers have many ways to get the schedules for a long period of time. For example, it can happen that a rolling stock schedule that is feasible for a given planning horizon cannot be immediately repeated for the next planning horizon due to maintenance requirements. As a result, the number of available units decreases. To remedy this, the number of units needed on certain future trips can be manually adjusted. With our approach, we give two different ways to generate the schedule for multiple planning horizons, as shown in Fig.10.

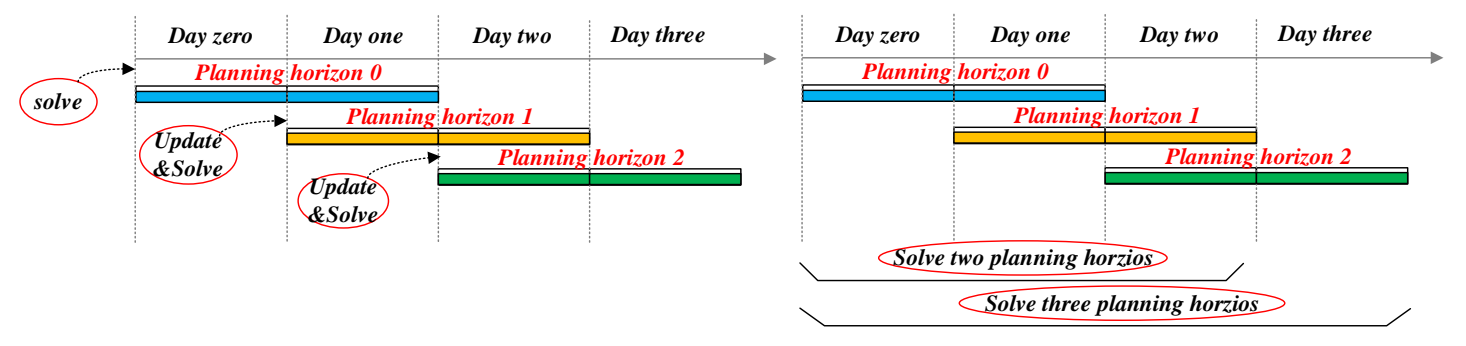

(a) rolling-horizon

(b) multiple-horizon

Fig. 10. Illustration of Calculating Multiple Planning Horizons

In Fig.10 (a) rolling-horizon, using the proposed algorithm we first generate a maintenance feasible rolling stock schedule for the first planning horizon (days zero and one). We can then update the accumulated mileage and running time of each unit, and find a maintenance feasible rolling stock schedule for days one and two. This rolling-horizon approach provides the possibility to generate schedules for any number of days. Following this 
way, we do the computation for the second planning horizon, named case M-2. Alternatively, as shown in Fig.10 (b), instead of repeatedly solving small planning horizons, we can try to solve multiple (more than two) days at once. The arc-flow model naturally increases in size due to the additional trips. In stage two, when generating trip sequences (which there are likely to be many more of) we check the maintenance requirements in each smaller planning horizon. In this work, we compare solving two planning horizons simultaneously (M-12) to two planning horizons solved sequentially (M-1 and M-2).

The dispatchers at the CHSR have validated the schedule we produce. Validation involved simulating the schedule in their timetabling software.

Information regarding the number of composition changes and the computation time (in seconds) for each case are given in Table 7. The columns "PH", "Coupling", "Decoupling" and "Sequences" refer to the planning horizon, number of couplings (outside the depot), number of (de) couplings (outside the depot), and the number of all possible trip sequences generated in stage 2. In all cases, there are no depot imbalances at the end of the planning horizon. However, some trip sequences originate and terminate at different depots. We term this as rolling stock "swap". It leads to the same number of units of a particular type being available at a certain depot, even though some units do not end up at where they started.

TABLE 7 Composition changes and computation time (in seconds) for different cases

\begin{tabular}{|c|c|c|c|c|c|c|c|}
\hline Data & $\overline{\mathrm{PH}}$ & Case & Coupling & Decoupling & Swaps & Sequences & Time \\
\hline \multirow{5}{*}{ Data-1 } & & $\overline{N M}$ & 2 & 0 & 16 & 53 & 106 \\
\hline & 1 & M & 2 & 0 & 16 & 53 & 106 \\
\hline & & M-D & - & - & - & 53 & 113 \\
\hline & 2 & M-2 & 2 & 0 & 10 & 53 & 117 \\
\hline & $1 \& 2$ & $\mathrm{M}-12$ & 2 & 0 & 14 & 656 & 360 \\
\hline \multirow{5}{*}{ Data-2 } & & NM & 2 & 0 & 0 & 67 & 207 \\
\hline & 1 & M & 2 & 0 & 0 & 67 & 207 \\
\hline & & M-D & - & - & - & 70 & 229 \\
\hline & 2 & M-2 & 1 & 0 & 0 & 67 & 231 \\
\hline & $1 \& 2$ & $\mathrm{M}-12$ & 2 & 2 & 0 & 1039 & 980 \\
\hline
\end{tabular}

From Table 7, we can see that the number of all possible individual trip sequences is not a large set for all of the cases, primarily because the solutions to the arc-flow model do not contain many composition changes. With the exception of case M-12 for Data-2, all cases can be solved within a few minutes. Also, as expected, it takes somewhat longer to solve two planning horizons simultaneously compared to solving two smaller planning horizons sequentially. In Table 8, we report some key characteristics of the solutions provided for each case.

TABLE 8 Key statistics for the different cases

\begin{tabular}{ccccccccccc}
\hline Data & PH & Case & RS & $\mathrm{L}_{\text {avg }}$ & $\mathrm{L}_{\min }$ & $\mathrm{L}_{\max }$ & $\mathrm{L}_{\text {tdh }}$ & $\mathrm{L}_{\text {adh }}$ & OP & Obj \\
\hline & & NM & 51 & $\mathbf{2 6 7 8 . 0}$ & 320 & 5419 & 3247 & 63.7 & 13 & 109727 \\
& 1 & $\boldsymbol{M}$ & $\mathbf{5 1}$ & $\mathbf{2 6 7 8 . 0}$ & $\mathbf{3 2 0}$ & $\mathbf{5 4 1 9}$ & $\mathbf{3 2 4 7}$ & $\mathbf{6 3 . 7}$ & $\mathbf{1 3}$ & $\mathbf{1 0 9 7 2 7}$ \\
& & $\mathrm{M}-\mathrm{D}$ & 53 & 2578.1 & 476 & 4771 & 3293 & 62.1 & 13 & 110593 \\
Data-1 & & $\mathrm{MA}$ & 57 & 2435 & 183 & 4898 & 5291 & 92.8 & 13 & 124471 \\
& ---1 & M-2 & 51 & 2678.2 & 327 & 5419 & 3255 & 63.8 & 13 & 109735 \\
& 2 & $\mathrm{MA}-2$ & 57 & 2435 & 183 & 4898 & 5291 & 92.8 & 13 & 124471 \\
& $-1 \& 2$ & M-12 & 65 & 4198.8 & 1912 & 7629 & 6448 & 99.2 & 26 & 219408 \\
\hline Data-2 & 1 & NM & 65 & 2760.7 & 660 & 5316 & 4736 & 72.9 & 18 & 145336 \\
\hline
\end{tabular}




\begin{tabular}{cccccccccc}
\hline & $\boldsymbol{M}$ & $\mathbf{6 5}$ & $\mathbf{2 7 6 0 . 7}$ & $\mathbf{6 6 0}$ & $\mathbf{5 3 1 6}$ & $\mathbf{4 7 3 6}$ & $\mathbf{7 2 . 9}$ & $\mathbf{1 8}$ & $\mathbf{1 4 6 0 3 6}$ \\
$\mathrm{M}-\mathrm{D}$ & 65 & 2764.5 & 543 & 5316 & 4762 & 73.3 & 18 & 146562 \\
$\mathrm{MA}$ & 70 & 2575.7 & 571 & 5229 & 5240 & 74.8 & 18 & 160753 \\
$-\mathrm{M}-2$ & 65 & 2760.8 & 631 & 5453 & 4728 & 72.7 & 18 & 146878 \\
-2 & MA-2 & 70 & 2575.7 & 571 & 5229 & 5240 & 74.8 & 18 & 160753 \\
$-1 \& 2$ & M-12 & 83 & 4223.2 & 1701 & 8527 & 9310 & 112.2 & 36 & 292610 \\
\hline
\end{tabular}

In Table 8, columns "RS", " $\mathrm{L}_{\text {avg }}$ ", " $\mathrm{L}_{\text {min }}$ ", " $\mathrm{L}_{\text {max }}$ ", " $\mathrm{L}_{\text {tdh }}$ " and " $\mathrm{L}_{\text {adh }}$ " refer to the number of rolling stock units used, the average running length $(\mathrm{km})$ of each unit, the minimal running length $(\mathrm{km})$ of a unit, the maximal running length $(\mathrm{km})$ of a unit, the total deadheading length $(\mathrm{km})$, the average deadheading length $(\mathrm{km})$ respectively. Column "OP" and "Obj" indicate the number of units overnighting at stations and total operating costs respectively.

At first, we focus on the schedules produced for the planning horizon one. For the cases $\mathrm{NM}$ and $\mathrm{M}$ of Data-1, the statistics in Table 8 are the same, which is to say the solution without maintenance (lower bound) is also the optimal solution considering maintenance. But when considering maintenance requirements in the second stage, the assignment for specific unit of the two case is different (e.g., they have different artificial costs and some rolling stock units violated the maintenance requirements in the schedule of case NM). In the rolling stock schedule without maintenance restrictions, 10 rolling stock units violate the level one maintenance rules by as much as $22.7 \%$ from a mileage perspective and $33.1 \%$ from a time perspective. For Data-2, we can see that the statistics of cases NM and M are quite similar. The case $\mathrm{M}$ costs $0.5 \%$ higher than case NM. Other than that, the statistics are the same. The reason for the differences in operating costs is the type of used rolling stock units, which can be seen in the assignment information produced by the second stage (e.g., the type of used rolling stock units in Table 9). The comparison of the two cases $M$ and NM indicates that the quality of the schedule in the case $M$ is very good, which reflects the effectiveness of our approach.

Looking at all of the cases for planning horizon one, it is clear from Table 8 that our approach can significantly reduce the deadhead mileage. In Data-1, comparing the two cases $\mathrm{M}$ and MA, the total deadhead mileage of case $\mathrm{M}$ is reduced by $2044 \mathrm{~km}(38.6 \%)$, and the average deadhead mileage of case $\mathrm{M}$ is reduced by $29.1 \mathrm{~km}$ (31.4\%). For Data-2, these decreases in percentages are $9.6 \%$ and $2.5 \%$, respectively. This is not as significant as Data-1, but it still shows a satisfactory impact. Reasons for the lower impact can be found in Table 5 and Table 7. Unlike Data-1, almost all rolling stock units are parked in the Zhengzhoudong depot in Data2, which causes no rolling stock swaps for Data-2, as shown in Table 7. Therefore, a more balanced parking distribution across the two depots may give rise to less deadhead mileage in Data-1. Overall, the total deadhead mileage gradually increases from case NM to case MA. Furthermore, the average rolling stock mileage is superior to that of the manual schedule. Both the reduction in deadhead mileage and the increase in rolling stock mileage help improve the utilization efficiency of the rolling stock units in the optimized schedule. In practice, the dispatchers always try to identify a schedule with long running distance and minimal deadhead mileage to make full use of the available rolling stock units.

Perhaps the most impressive statistic, however, is the reduction in operating costs. Maintaining and operating an HSR is not cheap, and the data in Table 8 show impressive results. The total cost of MA is the highest, and the total cost increases from case NM to case MA. When comparing case $\mathrm{M}$ with MA for Data 1 and Data 2, there is a reduction in the operating 
cost of $11.8 \%$ and $9.2 \%$. Even with the same operational rules as the manual schedule in case MA, M-D still shows $11.1 \%$ and $8.8 \%$ savings for the two datasets respectively. Another significant difference between the manual schedules and the optimized schedules is the decreased number of rolling stock units needed. In Data-1, compared with the case MA, the two cases $M$ and M-D can save six and four rolling stock units respectively. For Data-2, the two cases $M$ and M-D show a saving of five rolling stock units. The average cost of buying a new rolling stock unit in China is nearly $€ 10$ million. Furthermore, a reduction in the number of rolling stock units also reduces total operating costs, not to mention maintenance costs. The main reasons for the above difference are shown in Table 7 and from the basic information of rolling stock units used, which is presented in Table 9. In Table 9, the column UT represents the utilization of depots based on the number of rolling stock units used. Composition changes allow rolling stock units to be used more efficiently, but these can be difficult to identify when designing a schedule manually. We also utilize more rolling stock swaps than the manual solution. As mentioned earlier, unit type balance can be preserved without forcing individual rolling stock units to return to their starting point, which provides an additional level of flexibility.

TABLE 9. Basic information on rolling stock units used

\begin{tabular}{|c|c|c|c|c|c|c|c|c|}
\hline Data & $\mathrm{PH}$ & Case & Depot & CRH380AL & CRH380A & CRH380B & CRH6A & UT \\
\hline \multirow{14}{*}{ Data-1 } & \multirow{8}{*}{1} & \multirow{2}{*}{ NM } & $\mathrm{ZZ}$ & 0 & 0 & 11 & - & $61.1 \%$ \\
\hline & & & ZZD & 16 & 0 & 24 & - & $54.8 \%$ \\
\hline & & \multirow{2}{*}{$M$} & $Z Z$ & 0 & 0 & 11 & - & $61.1 \%$ \\
\hline & & & $Z Z D$ & 16 & 0 & 24 & - & $54.8 \%$ \\
\hline & & \multirow{2}{*}{ M-D } & $\mathrm{ZZ}$ & 0 & 1 & 11 & - & $66.72 \%$ \\
\hline & & & ZZD & 20 & 0 & 21 & - & $56.2 \%$ \\
\hline & & \multirow{2}{*}{ MA } & $\mathrm{ZZ}$ & 0 & 5 & 5 & - & $55.6 \%$ \\
\hline & & & ZZD & 22 & 4 & 21 & - & $64.4 \%$ \\
\hline & \multirow{4}{*}{2} & \multirow{2}{*}{ M-2 } & $\mathrm{ZZ}$ & 0 & 0 & 10 & - & $55.5 \%$ \\
\hline & & & ZZD & 26 & 0 & 15 & - & $56.2 \%$ \\
\hline & & \multirow{2}{*}{ MA-2 } & $\mathrm{ZZ}$ & 0 & 5 & 5 & - & $55.6 \%$ \\
\hline & & & ZZD & 22 & 4 & 21 & - & $64.4 \%$ \\
\hline & $18 ?$ & M 12 & $\mathrm{ZZ}$ & 0 & 0 & 11 & - & $61.1 \%$ \\
\hline & 102 & $101-12$ & ZZD & 26 & 0 & 28 & - & $73.9 \%$ \\
\hline \multirow{14}{*}{ Data-2 } & \multirow{8}{*}{1} & \multirow{2}{*}{ NM } & $\mathrm{ZZ}$ & - & - & - & 0 & 0 \\
\hline & & & ZZD & 26 & 0 & 39 & - & $72.2 \%$ \\
\hline & & \multirow{2}{*}{$M$} & $Z Z$ & - & - & - & 0 & 0 \\
\hline & & & $Z Z D$ & 26 & 2 & 37 & - & $72.2 \%$ \\
\hline & & \multirow{2}{*}{ M-D } & $\mathrm{ZZ}$ & - & - & - & 0 & $25 \%$ \\
\hline & & & ZZD & 24 & 2 & 39 & - & $72.2 \%$ \\
\hline & & \multirow{2}{*}{ MA } & $\mathrm{ZZ}$ & - & - & - & 7 & $87.5 \%$ \\
\hline & & & ZZD & 20 & 11 & 32 & - & $70 \%$ \\
\hline & \multirow{4}{*}{2} & \multirow{2}{*}{ M-2 } & $\mathrm{ZZ}$ & - & - & - & 8 & $100 \%$ \\
\hline & & & ZZD & 32 & 1 & 24 & - & $63.3 \%$ \\
\hline & & \multirow{2}{*}{ MA-2 } & $\mathrm{ZZ}$ & - & - & - & 7 & $87.5 \%$ \\
\hline & & & ZZD & 20 & 11 & 32 & - & $70 \%$ \\
\hline & \multirow{2}{*}{$1 \& 2$} & \multirow{2}{*}{ M-12 } & $\mathrm{ZZ}$ & - & - & - & 8 & $100 \%$ \\
\hline & & & ZZD & 34 & 2 & 39 & - & $83.3 \%$ \\
\hline
\end{tabular}

Compared to the dispatcher's schedule, we can see that the rolling stock units of type CRH380B are favored by the optimized schedule. This is because the operating costs of type CRH380B are fewer than other unit types, and thus the optimized approach naturally exploits 
this. Table 9 also shows the occupancy of two depots under different rolling stock schedules. We choose the cases M, M-D and MA for the two datasets to compare the running mileage of the individual units, which are illustrated in Fig. 11.

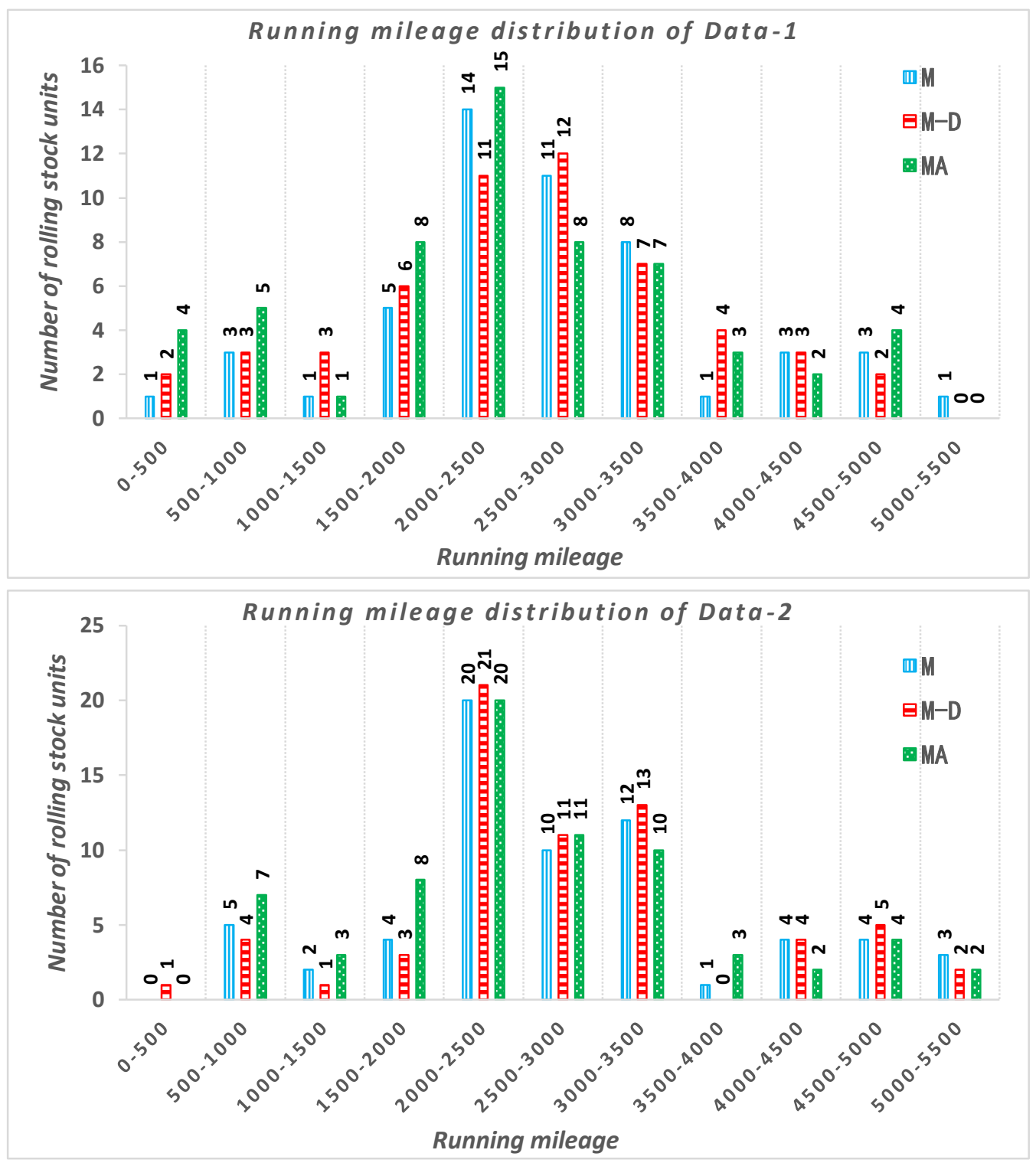

Fig. 11 Rolling stock running mileage distribution

As shown in Fig. 11, for both datasets, the running mileages of the individual units in the optimized schedule are typically longer and have less variation. One possible reason for this is that dispatchers will normally use fixed rolling stock units to complete some short-distance trips, while the optimized approach can find long-distance trips to merge with short-distance trips.

The schedules for planning horizon two use the same number of rolling stock units as the schedules of planning horizon one for both datasets. The optimized schedule still has a significant advantage over the manual schedule. Comparing the information of the two cases M and M-2 in Table 9, it is not difficult to see that the types of rolling stock units used have changed and the number of other rolling stock unit types (except for type CRH380B) increases in the case $\mathrm{M}-2$. The reason for this is that the overnighting rolling stock units are replaced by 
some other rolling stock unit types.

The number of used rolling stock units during two planning horizons obtained from different cases are shown in Fig.12. For both datasets, from Table 8 and Fig.12, we can see that statistics of case M-12 and cases [M, M-2] (represents the schedule of two planning horizon through the rolling-horizon way) are very similar. Case M-12 is slightly better than cases [M, M-12]. Compared to case M-12, the operating costs of cases [M, M-2] is $0.1 \%$ and $0.02 \%$ higher for two datasets, respectively. In addition, both of the case M-12 and cases [M, M-2] are far better than the manual cases [MA, MA-2].

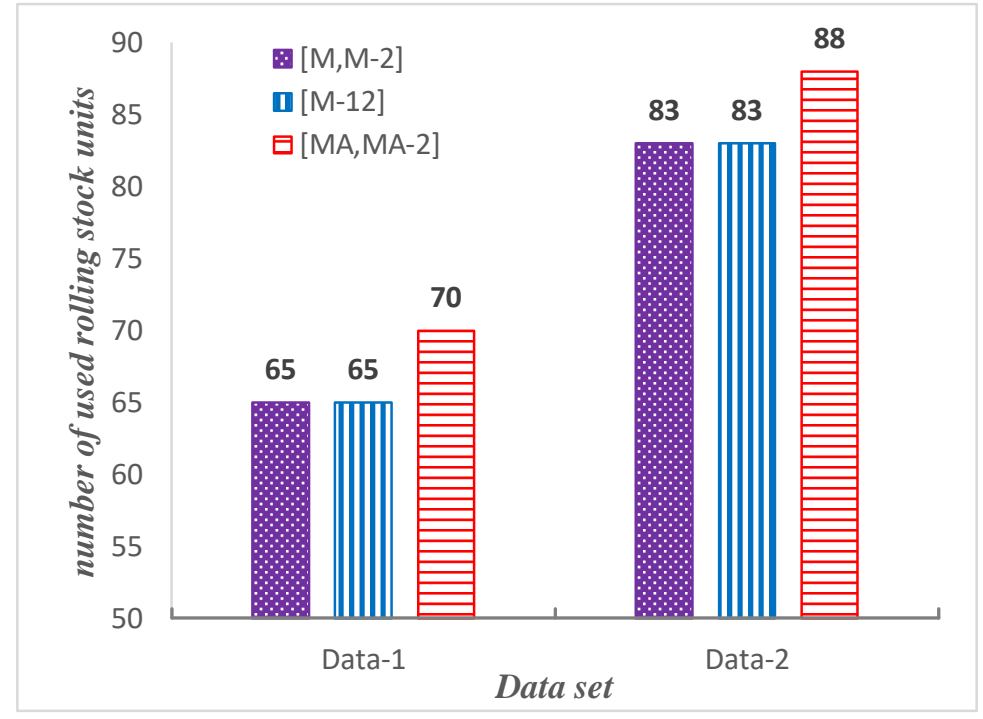

Fig.12 The number of used rolling stock units

The schedule obtained by rolling-horizon is actually a feasible solution of multiple-horizon, which is to say that if there is no consideration of computation time or the memory usage, the multiple-horizon can produce a schedule which is at least the same as rolling-horizon or better.

\subsection{Method Scalability}

The datasets considered in the computational study of Section 5.2 only include two depots, which naturally limits the number of locations where (de) coupling can occur and the number of possible paths in the path enumeration stage of the algorithm. To investigate the scalability of the method we therefore consider several artificial instances in which we vary the number of depots available. After discussion with the dispatchers in Zhengzhou Railway, five alternative depot locations were introduced. Based on the importance of the new depots, we consider five different cases with one [Shangqiu], two [Shangqiu, Luoyanglongmen], three [Shangqiu, Luoyanglongmen, Xuchangdong], four [Shangqiu, Luoyanglongmen, Xuchangdong, Anyangdong], and five [Shangqiu, Luoyanglongmen, Xuchangdong, Anyangdong, Jiaozuo] additional depots. Each of the new depots is assumed to be $10 \mathrm{~km}$ from its corresponding station. Therefore, a total of seven depots in the network, which account for approximately $20 \%$ of the total number of stations, is available for composition changes. We further assume that maintenance can be performed at any of the new depots, and that all units are assumed to be located at either of the two existing depots. Unlike "Zhengzhoudong" and "Zhengzhou" depots, only units which are available at the new depots can be used for composition changes during the operating period (i.e., the deadheading of units for composition changes is not permitted). 
The results for Data-1 and Data-2 using the proposed two-stage approach for one planning horizon are given in Table 10. For each instance, Table 10 reports the number of available depots "Depot_num", the number of (de) couplings, the number of swaps, the number of units needed, and the computational time (in seconds).

TABLE 10 Composition changes and computation time (in seconds) for new cases

\begin{tabular}{cccccccc}
\hline Data & Index & Depot $_{\text {num }}$ & Coupling & Decoupling & Swaps & Sequences & Time \\
\hline \multirow{5}{*}{ Data-1 } & 1 & 3 & 1 & 0 & 14 & 53 & 106 \\
& 2 & 4 & 1 & 0 & 10 & 53 & 128 \\
& 3 & 5 & 1 & 0 & 12 & 53 & 134 \\
& 4 & 6 & 1 & 0 & 8 & 53 & 159 \\
& 5 & 7 & 1 & 0 & 14 & 53 & 167 \\
\hline \multirow{5}{*}{ Data-2 } & 6 & 3 & 2 & 0 & 0 & 69 & 223 \\
& 7 & 4 & 2 & 0 & 0 & 69 & 254 \\
& 9 & 5 & 2 & 0 & 0 & 69 & 293 \\
& 9 & 6 & 2 & 0 & 0 & 69 & 386 \\
\end{tabular}

Compared to Table 7, one can observe that the computation times increase as the number of depots increase; however, the run times still remain satisfactory. The number of units needed in these cases is essentially the same; the maximum number of units is 53 for Data- 1 and 69 for Data-2. The number of (de) couplings does not change greatly with the addition of more depots. The introduction of the new depots does, however, provide greater flexibility with respect to the maintenance requirements and the dead heading possibilities. This is confirmed by the key statistics of these instances in Table 11.

TABLE 11 Key statistics for new cases

\begin{tabular}{ccccccccccc}
\hline Data & Index & Depot $_{\text {num }}$ & $\mathrm{RS}$ & $\mathrm{L}_{\text {avg }}$ & $\mathrm{L}_{\min }$ & $\mathrm{L}_{\max }$ & $\mathrm{L}_{\text {tdh }}$ & $\mathrm{L}_{\text {adh }}$ & $\mathrm{OP}$ & Obj $^{\text {Data-1 }}$ \\
& 1 & 3 & 53 & 2593.1 & 420 & 5233 & 4095 & 77.3 & 11 & 108735 \\
& 2 & 4 & 53 & 2590.5 & 464 & 5206 & 3959 & 74.7 & 11 & 106999 \\
& 3 & 5 & 53 & 2590.4 & 442 & 5336 & 3952 & 74.6 & 11 & 106992 \\
& 4 & 6 & 53 & 2590.3 & 410 & 5206 & 3949 & 74.5 & 11 & 106989 \\
& 5 & 7 & 53 & 2590.3 & 397 & 5206 & 3949 & 74.5 & 11 & 106989 \\
\hline \multirow{5}{*}{ Data-2 } & 6 & 3 & 67 & 2691.2 & 510 & 5316 & 5594 & 88.5 & 16 & 144654 \\
& 7 & 4 & 67 & 2689.2 & 631 & 5316 & 5459 & 81.5 & 16 & 142919 \\
& 8 & 5 & 67 & 2689.0 & 631 & 5037 & 5445 & 81.3 & 16 & 142905 \\
& 9 & 6 & 67 & 2688.9 & 616 & 5316 & 5439 & 81.2 & 16 & 142899 \\
& 10 & 7 & 67 & 2688.9 & 822 & 5316 & 5439 & 81.2 & 16 & 142899 \\
\hline
\end{tabular}

Table 11 indicates that smaller objective values are obtained when increasing the number of available depots (although the improvement steadily decreases). The number of units overnighting also decreases as units can now be deadheaded on the day of operation from one of the closer depot stations instead of overnighting. As a result, the total number of units needed increases. This is due to the fact that trips that were previously served by reusing the overnighting units are now served by other units. Since all units are parked in the original two depots, the deadheading from one of the two original depots to the new depot stations leads to a slight increase in deadhead mileage. Overall, the benefits of reducing overnight penalties are greater than the losses caused by increased deadhead mileage. It is also apparent that there is 
only a very small improvement in the objective function when the number of additional new depots reaches four. Furthermore, when the number of available depots increases from six to seven, the objective value remains unchanged in both cases.

The number of depots definitely provides more flexibility with respect to composition changes and maintenance feasibility; however, simply providing more locations for (de) coupling activities does not necessarily induce a better objective value. The connectivity of the underlying railway network and timetable structure may also have significant impact on the optimization results. Here, connectivity refers to how many stations, on average, are directly connected to any other stations. High speed railway networks typically have a low connectivity. We therefore expect the proposed methodology to perform well on networks with a similar structure to the one considered here. We also expect the performance of the method to deteriorate as the connectivity of the network increases. A detailed study of this, however, remains a topic for future work.

\section{Conclusion}

At the CHSR, rolling stock scheduling with maintenance requirements is a complex and important problem. This problem has mainly been solved manually by dispatchers. In this paper, we present a two-stage approach for solving the rolling stock scheduling problem with maintenance requirements for a busy part of the CHSR network. Two MILP problems form the core of our approach: one generates a set of candidate rolling stock schedules ignoring maintenance requirements, and the other one assesses rolling stock schedules for maintenance feasibility. Real-world instances provided by the Zhengzhou HSR network have been used to test the performance of the proposed approach. The paper also provides a clear overview of the operational processes at the CHSR, and how they differ from processes at European railways.

Dispatchers try to efficiently piece together feasible trip sequences. Numerical results from the test cases show that for a two to three day planning horizon enumerated set of trip sequences is generally not very large for a given rolling stock schedule. This is not surprising as there are a limited number of places in the network where compositions can be changed. However, the results also show that only a small number of selected composition changes are needed to significantly reduce the operational cost, up to $12 \%$. Furthermore, the schedules that we obtain turn out to be more efficient in terms of deadhead mileage and running time. The computation time of our approach is trivial, which typically takes a few minutes in total.

This paper highlights a new approach designed for an important and practical problem. The applicability of the method in practice has been an important aspect of the work. The project has therefore been carried out in close collaboration with dispatchers at the Zhengzhou Group, where all of the schedules have been verified as implementable by the dispatchers.

Several directions for further research exist. Different timetables exist for some special occasions, such as the Chinese New Year. Typically, these special occasions are busier periods, and it would be interesting to benchmark the performance of the approach against such instances. We would also like to compare the performance of the proposed approach with existing algorithms for this problem, and, hopefully, simultaneously provide a comparison of different algorithms across different railway companies (Chinese and European). Finally, the CHSR is considering building new depots throughout the network and it is interested in the 
potential impact of different depot locations on the quality of the maintenance feasible rolling stock schedules. The current framework can be easily extended to handle this.

\section{Acknowledgements}

This work was supported by the National Key R\&D Program [No. 2017YFB1200700] and National Nature Science Foundation of China [No. 71871188, No. U1834209]. The first and fourth author gratefully acknowledges the financial support from the China Scholarship Council [201707000041, 20170700008]. Also, the authors would like to thank the China Zhengzhou Railway company for providing valuable advice and test data.

\section{References}

Wangshu Luo (2018, January, 3) Rail system to grow by 4,000 km in 2018, China Daily, http://www.chinadaily.com.cn/a/201801/03/WS5a4bfb27a31008cf16da4b5c.html, accessed on $12 / 10 / 2018$.

Abbink, E., Berg, B.v.d., Kroon, L., Salomon, M., 2004. Allocation of railway rolling stock for passenger trains. Transportation Science 38 (1), 33-41.

Alfieri, A., Groot, R., Kroon, L., Schrijver, A., 2006. Efficient circulation of railway rolling stock. Transportation Science 40 (3), 378-391.

Brucker, P., Hurink, J., Rolfes, T., 2003. Routing of railway carriages. Journal of Global Optimization 27 (2), 313-332.

Borndörfer, R., Reuther, M., Schlechte, T., Waas, K., Weider, S., 2016. Integrated optimization of rolling stock rotations for intercity railways. Transportation Science 50 (3), 863-877

Cordeau, J.-F., Soumis, F., Desrosiers, J., 2001. Simultaneous assignment of locomotives and cars to passenger trains. Operations Research 49 (4), 531-548.

Cacchiani, V., Caprara, A., \& Toth, P., 2013. A Lagrangian heuristic for a train-unit assignment problem. Discrete Applied Mathematics, 161(12), 1707-1718. https://doi.org/10.1016/j.dam.2011.10.035

Fioole, P.-J., Kroon, L., Maróti, G., Schrijver, A., 2006. A rolling stock circulation model for combining and splitting of passenger trains. European Journal of Operational Research 174 (2), 1281-1297.

Haahr, J. T., Wagenaar, J. C., Veelenturf, L. P., \& Kroon, L. G., 2016. A comparison of two exact methods for passenger railway rolling stock (re)scheduling. Transportation Research Part E Logistics \& Transportation Review, 91, 15-32.

Jean-François Cordeau. 2000. A Benders decomposition approach for the locomotive and car assignment problem. Transportation Science, 34:133-149.

Lusby, R. M., Larsen, J., Ehrgott, M., \& Ryan, D. 2011. Railway track allocation: models and methods. Or Spectrum, 33(4), 843-883.

Lusby, R. M., Haahr, J. T., Larsen, J., \& Pisinger, D., 2017. A branch-and-price algorithm for railway rolling stock rescheduling. Transportation Research Part B Methodological, 99, 228250.

Li, Y., Miao, Q., \& Wang, X. B., 2014. High-Speed Train Network Routing with Column Generation. Transportation Research Record, 2466(2466), 58-67. 
https://doi.org/10.3141/2466-07

Lai, Y.-C., Fan, D.-C., Huang, K.-L., 2015. Optimizing rolling stock assignment and maintenance plan for passenger railway operations. Computers \& Industrial Engineering 85 (Supplement C), 284-295.

Lai, Y.-C.R., Wang, S.-W., Huang, K.-L., 2017. Optimized train-set rostering plan for Taiwan HighSpeed Rail. IEEE Transactions on Automation Science and Engineering 14 (1), 286-298. Maróti, G., Kroon, L., 2005. Maintenance routing for train units: the transition model. Transportation Science 39 (4), 518-525.

Gábor Maróti and Leo Kroon., 2007. Maintenance routing for train units: The interchange model. Computers \& Operations Research, 34(4):1121-1140.

Nishi, T., Ohno, A., Inuiguchi, M., Takahashi, S., Ueda, K., 2017. A Combined column generation and heuristics for railway short-term rolling stock planning with regular inspection constraints. Computers \& Operations Research 81, 14-25.

Nielsen, L. K. Maróti, G., Kroon, L., 2012. A rolling horizon approach for disruption management of railway rolling stock. European Journal of Operational Research, 220(2), 496509.

Ohno, A., Nishi, T., Inuiguchi, M., Takahashi, S., \& Ueda, K., 2012. Application of column generation for train-set scheduling problems with regular maintenance constraints. Ieej Transactions on Electronics, Information and Systems, 132(1), 151-159+17, 17-159. https://doi.org/10.1541/ieejeiss.132.151

Peeters, M., Kroon, L., 2008. Circulation of railway rolling stock: a branch-and-price approach. Computers \& Operations Research 35 (2), 538-556.

Thorlacius, P., Larsen, J., \& Laumanns, M., 2015. An integrated rolling stock planning model for the Copenhagen suburban passenger railway. Journal of Rail Transport Planning and Management, 5(4), 240-262. https://doi.org/10.1016/j.jrtpm.2015.11.001

Wang, Y., Liu, J., Miao, J., 2010. Column generation algorithms based optimization method for maintenance scheduling of multiple units. China Railway Science 31 (2), 115-120.

Wagenaar, J.C., Kroon, L.G. and Schmidt, M., 2017. Maintenance appointments in railway rolling stock rescheduling. Transportation Science, 51(4), pp.1138-1160.

Ziarati, K., Soumis, F., Desrosiers, J., Gélinas, S., Saintonge, A., 1997. Locomotive assignment with heterogeneous consists at CN North America. European Journal of Operational Research 97 (2), 281-292. 